\title{
Towards Energy-Fairness for Broadcast Scheduling with Minimum Delay in Low-Duty-Cycle Sensor Networks
}

\author{
Lijie $\mathrm{Xu}^{1,2}$, Xiaojun $\mathrm{Zhu}^{3}$, Haipeng $\mathrm{Dai}^{2}$, Xiaobing $\mathrm{Wu}^{2}$, Guihai Chen ${ }^{2,4}$ \\ ${ }^{1}$ School of Computer Science and Technology, Nanjing University of Posts and \\ Telecommunications, Nanjing 210023, China \\ ${ }^{2}$ State Key Laboratory for Novel Software Technology, Nanjing University, Nanjing, China \\ ${ }^{3}$ College of Computer Science and Technology, Nanjing University of Aeronautics and \\ Astronautics, Nanjing, China \\ ${ }^{4}$ Shanghai Key Laboratory of Scalable Computing and Systems, Shanghai Jiao Tong \\ University, Shanghai, China
}

\begin{abstract}
Broadcast scheduling for low-duty-cycle wireless sensor networks (WSNs) has been extensively studied recently. However, existing solutions mainly focused on optimizing delay and (or) total energy consumption without considering load distribution among nodes. Due to limited energy supply for sensor nodes, heavily loaded sensors often run out of energy quickly, reducing the lifetime of the whole network. In this paper, we target at minimizing the maximum transmission load of a broadcast schedule for low-duty-cycle WSNs, subject to the constraint that each node should have the minimum end-to-end delay under the broadcast schedule. We prove that it is NP-hard to find the optimal schedule. Then, we devise a Load-Balanced Parents Assignment Algorithm (LBPA-A) that achieves $\lambda$-approximation ratio, where $\lambda$ denotes the maximum number of neighbors that are scheduled to wake up at the same time and is typically a small number in low-duty-cycle WSNs. Further, we introduce how to solve this problem in a distributed manner. Our simulation results reveal that compared with the traditional solutions, our proposed LBPA-A and distributed solution
\end{abstract}

\footnotetext{
Email addresses: ljxu83@gmail.com (Lijie $\mathrm{Xu}^{1,2}$ ), xzhu@nuaa.edu.cn (Xiaojun $\mathrm{Zhu}^{3}$ ), dhpphd2003@gmail.com (Haipeng Dai ${ }^{2}$ ), wuxb@nju.edu.cn (Xiaobing $\mathrm{Wu}^{2}$ ), gchen@nju.edu.cn (Guihai Chen ${ }^{2,4}$ )

Preprint submitted to Computer Communications

January 21, 2015
}

(C) 2015. This manuscript version is made available under the Elsevier user license http://www.elsevier.com/open-access/userlicense/1.0/ 
both exhibit much better average performance in terms of energy-fairness, total energy consumption and delivery ratio.

Keywords: wireless sensor networks; low-duty-cycle; broadcast scheduling; load balancing; energy efficient.

\section{Introduction}

As an important fundamental function, multi-hop broadcasting in wireless sensor networks (WSNs) has been extensively studied in the past few years. Many applications in WSNs, e.g., environmental monitoring, medical care system and scientific exploration, require that the sink node should disseminate the system configurations to all sensor nodes in a timely and energy-efficient manner. In practice, it has been verified [1] that idle listening is the major source of energy waste in WSNs. As shown in [2], the commonly adopted ChipCon CC2420 radio draws $18.8 \mathrm{~mA}$ at receiving mode or idle listening mode, and draws $17.4 \mathrm{~mA}$ at sending mode, which implies that idle listening actually consumes approximately the same amount of power as in receiving and sending mode. In order to significantly reduce the energy waste caused by idle listening, sensor nodes are often put in a low-duty-cycle mode where every sensor node has its own working schedule to alternate periods of work with sleep.

How to optimize the energy efficiency of broadcasting in low-duty-cycle WSNs has been well-investigated by many existing works, in which the proposed solutions can achieve high energy efficiency in terms of total energy consumption. However, it may not be appropriate to take total energy consumption as the main metric to characterize energy efficiency in sensor networks. As we know, it is typically hard to replace or recharge batteries for sensor nodes as many WSNs are deployed in a tough environment that human beings are not easy to access to. This fact implies it is more important for sensor networks to take energy-fairness as the first concern, since unbalanced load could make nodes with heavy workload deplete their energy much faster so that the network is disabled earlier, e.g., the monitoring field cannot be fully covered, or the network 
becomes disconnected. Currently, most of the existing works mainly pay attention to load-balanced data collection in low-duty-cycle WSNs. However, very few of them consider load balancing of data dissemination, which is an important function in sensor networks. In low-duty-cycle WSNs, one-hop broadcasting is usually implemented by multiple unicasts. Once the broadcasting schedule is established, it is normally performed for a rather long duration and is unlikely to be updated frequently due to high update overhead. Therefore, a broadcasting schedule without careful design may lead to highly unbalanced broadcasting load among sensor nodes. As the number of broadcasting increases, this unbalancing will be further intensified. For low-duty-cycle WSNs where load-balanced data collection are adopted, designing a broadcasting schedule to guarantee energy fairness among sensor nodes is an important issue.

Compared with always-awake networks, low-duty-cycle sensor networks usually yield a notable increase on communication delay due to the periodic sleeping, and thus delay is always taken as the first consideration in such networks. In many broadcasting applications without cooperation requirement such as configuration dissemination, each node is expected to receive the broadcasting message as soon as possible to update the configuration so that the new system requirement can be satisfied in a short period of time. In other words, the end-to-end (E2E) delay from the sink node to each sensing node is desired to be minimized for broadcasting in such applications. For example, GreenOrbs is a consistently operating sensor network system deployed on Tianmu mountain of China for the aim of forest monitoring. It periodically collects various sensory data including temperature, humidity, illumination, and carbon dioxide titer. The collected information is utilized to support various significant applications, such as forest surveillance, forestry observation and research, fire risk evaluation, and succor in the wild. Occasionally, we need to change the sampling period or other parameters of each node. In this case, the sink node needs to broadcast the updated configuration in the network as soon as possible so that the new system requirement can be satisfied as soon as possible. As for another example, alarm detection system is also a type of widely used applications for WSNs. 
Upon detecting that a parameter (e.g., temperature, and humidity) is above or below some threshold, the sensor node will report it to the sink quickly so that a prompt action can be taken. For this kind of applications, we sometimes need to change the system requirement (e.g., to change the alarm threshold), and the sink needs to broadcast the message attached with the updated alarm threshold in the network as soon as possible so that the chance of false positive or false negative can be reduced as much as possible. To this end, we identify the minimum E2E delay as a firm requirement, and thus our optimization goal becomes balancing the energy consumption among nodes while guaranteeing the minimum E2E delay.

In this paper, we focus on the Load-Balanced Minimum E2E-delay Broadcast Scheduling Problem (LB-MEBS), namely how to minimize the maximum transmission load of a broadcasting schedule for low-duty-cycle WSNs, subject to the constraint that each node should have the minimum end-to-end delay under the broadcasting schedule.

The main contributions of this work are as follows:

- To the best of our knowledge, we are the first to investigate the loadbalanced minimum delay broadcast scheduling problem for low-duty-cycle WSNs. We transform our problem into the equivalent Load-Balanced Parents Assignment Problem (LBPA), and prove its NP-hardness.

- We propose the Load-Balanced Parents Assignment Algorithm (LBPAA) to tackle the LBPA problem, and show that LBPA-A can achieve $\lambda$ approximation, where $\lambda$ denotes the maximum number of neighbors that are scheduled to wake up at the same time and is typically a small number in low-duty-cycle WSNs.

- We also propose an efficient distributed solution, i.e., DLBPA-A, to solve our problem. The message complexity of this algorithm is $O\left(N^{2}+N \cdot d_{\max }^{2}\right)$ where $N$ and $d_{\max }$ denote the number of nodes and the maximum node degree in the network, respectively. 
- Our simulation results reveal that compared with the traditional solutions, LBPA-A and DLBPA-A both exhibit much better average performance in terms of energy-fairness, total energy consumption and delivery ratio.

The rest of the paper is organized as follows: Section 2 summarizes the related work. Section 3 illustrates the network model and formulates the problem. Section 4 analyzes the problem hardness. Detailed description and analysis of our proposed algorithm are presented in Section 5. Section 6 introduces an efficient distributed solution, followed by the discussion about practical issues and the simulation results in Section 7 and Section 8. Section 9 concludes our findings.

\section{Related Work}

In recent years, a number of works that focus on energy-efficient broadcast scheduling problem in low-duty-cycle WSNs have been proposed $[3,4,5,6,7,8$, 9, 10, 11, 12, 13]. Hong et al. [4] studied the Minimum-Transmission Broadcast problem in uncoordinated duty-cycled networks and proved its NP-hardness. They proposed a centralized approximation algorithm with a logarithmic approximation ratio and a distributed approximation algorithm with a constant approximation ratio for this problem. In [6], the authors considered link correlation and devised a novel flooding scheme to reduce energy consumption of broadcasting by letting nodes with high correlation be assigned to a common sender. Xu et al. [7] utilized the broadcasting spatiotemporal locality to address the latency-optimal minimum energy broadcast problem in low-duty-cycle WSNs. In [10], the authors studied the duty-cycle-aware Minimum-Energy Multicasting problem in WSNs both for one-to-many multicasting and for all-to-all multicasting. Han et al. [11] studied the problem of minimizing the expected total transmission power for reliable data dissemination in duty-cycled WSNs. Due to the NP-hardness of the problem, they designed efficient approximation algorithms with provable performance bounds for it. Cheng et al. [12] proposed a novel dynamic switching-based reliable flooding (DSRF) framework, which is 
designed as an enhancement layer to provide efficient and reliable delivery for a variety of existing flooding tree structures in low duty-cycle WSNs. In [13], the authors investigated the energy efficient broadcast problem with minimum latency constraint in low-duty-cycle WSNs with unreliable links, and proposed a distributed heuristic solution to tackle this problem. However, all of these works mainly focus on the minimization of total energy consumption rather than load balancing. Recently, Glossy broadcasting scheme [14] has gained much attraction amongst researchers, as it uses constructive interference for flooding and thus eliminates any need to establish a schedule for broadcasting. However, it mainly focuses on the latency and reliability of broadcasting but not the load balance problem.

Currently, load balancing for applications of data collection in sensor networks has also been extensively investigated by many works $[15,16,17,18$, 19, 20, 21, 22]. Wu et al. [15] proposed a novel nonuniform node distribution strategy to achieve nearly balanced energy depletion in the network. Jurdak et al. [16] proposed a cross-layer framework to balance the load in sensor networks via greedy local decisions. Xiong et al. [17] studied the multiple task scheduling problem for low-duty-cycle WSNs. They presented several efficient scheduling algorithms to achieve load balancing among sensor nodes in both spatial and temporal dimensions. In [18], the authors developed a delay-constrained data aggregation scheme for duty cycle sensor networks to balance the nodal lifetime of all nodes. Besides, some works such as [19] also consider employing a mobile sink or mobile relay to collect data so as to balance loads among sensor nodes. In [20], the authors investigated the problem of controlling node sleep intervals so as to achieve the min-max energy fairness in asynchronous dutycycling sensor networks, the proposed algorithm is self-adjustable to the traffic load variance and is able to serve as a unified framework for a variety of asynchronous duty-cycling MAC protocols. However, to the best of our knowledge, none of the existing works consider load balancing of broadcasting applications for sensor networks under low-duty-cycle operation, which is an important issue for those networks with load balanced data collection. 


\section{Network Model and Problem Statement}

\subsection{Network Model and Assumptions}

We assume that time is divided into a number of equal time slots and each time slot is set long enough to accommodate at least one packet transmission. The network is a multi-hop network, and for energy conservation, it is assumed to be put in a low-duty-cycle mode. In such mode, each sensor node alternates between sleep state and active state according to its predefined working schedule. Such working schedule depends on a particular power management protocol, e.g., [23]. When staying in sleep state, any node will turn all its function modules off except a timer to wake itself up, and in active state, it will turn on its radio and keep awake. An active node is able to sense an event, transmit a packet or receive a packet. We assume that working schedule is periodic and all sensor nodes have the same period length of working schedule. For simplicity and without loss of generality, we suppose that one period of any node's working schedule consists of $L$ time slots where only one time slot is in active state and the others are in sleep states. Here, the working schedule of any node $j$ is denoted by the set $T_{s}(j)=\left\{i \mid i \in\{0, \ldots, L-1\}\right.$ and $\left.\tau_{j}^{i}=1\right\}$, in which $\tau_{j}^{i}=1$ denotes the time slot $i$ for each schedule period of node $j$ is in active state. Fig. 1 illustrates an example of the periodic working schedule $\{2\}$, where $L=5$ and the node only keeps awake at time slot 2 for each period of working schedule.

In this paper, we use the undirected spatiotemporal topology graph $G=$ $(V, E, W, L)$ to represent the network topology and nodes' working schedules, where $V$ represents the set of $N$ nodes including the sink node $v_{0}$ and all sensing nodes $\left\{v_{1}, v_{2}, \ldots, v_{N-1}\right\}, E$ represents the set of all communication links, $W$ denotes the set of working schedules for all nodes, and $L$ denotes the schedule period length of each node. Note that, our solution is also applicable to the general case where one period of any node's working schedule contains one or more than one active state time slot.

As the same with most of the literature for low-duty-cycle WSNs (e.g., [3, 4, $5,7,10,11,12,13,24,25,26,27])$, we assume that 1) time synchronization is 
achieved; 2) each node is aware of the working schedules of all its neighboring nodes; and 3) each node can transmit its packets at any time but can only receive packets from its neighbors in active states. Specifically, if any sender wants to send a message to its receiver, it will set a timer to wake up itself at the beginning of the receiver's next active state to finish the transmission. For simplicity, we do not consider the packet collision problem in our model, and this practical issue and its efficient solution will be discussed in Section 8. Besides, we make a practical power consumption assumption, that is, idle listening consumes approximately the same amount of power as in receiving and sending mode, which has been shown in [2].

In this paper, we also assume that at any time the network is processing at most one request, i.e., two successive broadcast requests of the sink do never overlap in the network. This assumption is always true in practice since the broadcasting originated from the sink (e.g., configuration dissemination) is usually a relatively infrequent event in real sensor network systems, which implies the time duration between any two successive broadcast requests is usually long enough (much longer than the minimum broadcast latency) for real broadcast applications. Specifically, the sink in real broadcast applications will usually not send a request (i.e., configuration update command) until the last sent request is received by all the nodes in the network and the new updated configuration at each node is running for a duration (i.e., the new updated system requirement is satisfied for a duration). Therefore, we can consider broadcast request one by one without overlapping, in other words, we only need to focus on one broadcast request in the paper.

A summary of the primary notations in this paper is given in Table 1. 
Table 1: Summary of the primary notations

\begin{tabular}{r|l}
\hline Symbol & Meaning \\
\hline \hline$T_{s}(v)$ & The set of active time slots of node $v$ \\
$d_{i j}$ & Point-to-point transmission delay from node $v_{i}$ to $v_{j}$ \\
delay $(u, v)$ & Minimum end-to-end delay from node $u$ to node $v$ \\
$\operatorname{Load}(v)$ & Transmission load of node $v$ \\
$d e g(v)$ & Degree of node $v$ \\
$C P S(v)$ & Candidate parents set of node $v$ \\
$C C S(v)$ & Candidate children set of node $v$ \\
$\operatorname{Load}_{E}(v)$ & Transmission load of node $v$ in the edge set $E$ \\
$\operatorname{deg}_{E}(v)$ & Degree of node $v$ in the edge set $E$ \\
$C P S_{E}(v)$ & Candidate parents set of node $v$ in the edge set $E$ \\
$C C S_{E}(v)$ & Candidate children set of node $v$ in the edge set $E$ \\
$S(v, t)$ & The set of message receivers in $C C S(v)$ if node $v$ \\
$T R(v, t)$ & sends a message at tine slot $t$ \\
$R D_{E}(v, t)$ & The transmission of node $v$ at time slot $t$ \\
$T S(v)$ & The redundancy degree of the transmission $T R(v, t)$ \\
$L N S(v)$ & Transmission strategy set of node $v$ \\
\hline
\end{tabular}

\subsection{Problem Statement}

Definition 1 (Transmission Load). Suppose node $v_{i}$ needs to send a message to all nodes in a subset $N\left(v_{i}\right)$ of its neighbors. We define its transmission load $\operatorname{Load}\left(v_{i}\right)$ as the number of additional slots that $v_{i}$ must be awake to finish the transmission, where an additional slot of $v_{i}$ is a slot originally not scheduled to be awake. That is,

$$
\operatorname{Load}\left(v_{i}\right)=\left|\bigcup_{v_{j} \in N\left(v_{i}\right)} T_{s}\left(v_{j}\right)-T_{s}\left(v_{i}\right)\right|
$$

where $T_{s}(v)$ denotes the set of active time slots of node $v$.

Here, we use an example, as shown in Fig. 2 with $L=10$, to illustrate the above definition. In Fig. 2(a), suppose that the sender $v_{0}$ wants to broadcast a packet to all the receivers in $\left\{v_{1}, \ldots, v_{5}\right\}$. We can find that none of the receivers has the same working schedule with the sender, and sender $v_{0}$ has to additionally wake up and transmit the packet at time slots $3,5,6$ and 8 so as to finish the one-hop broadcasting, so the transmission load $\operatorname{Load}\left(v_{0}\right)=$ $|\{3,5,6,8\}-\{2\}|=4$ in this case. In Fig. 2(b), node $v_{2}$ has the same working 


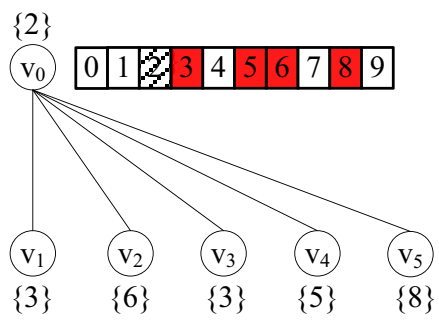

(a) Case I: $\operatorname{Load}\left(v_{0}\right)=4$

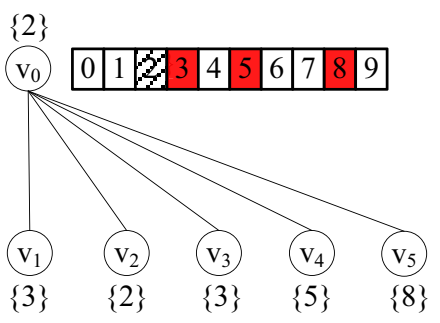

(b) Case II: $\operatorname{Load}\left(v_{0}\right)=3$

Figure 2: Illustration of transmission load

schedule $\{2\}$ with the sender $v_{0}$, which implies $v_{0}$ will transmit the packet to $v_{2}$ at its scheduled time slot and thus the transmission energy from $v_{0}$ to $v_{2}$ can be approximately neglected since in practice, idle listening mode has nearly the same energy consumption rate with the sending mode [2]. Accordingly, in Fig. 2 (b), the broadcasting energy consumption of sender $v_{0}$ mainly comes from the transmissions at 3 non-scheduled additional time slots (i.e., time slots 3, 5 and 8), so the transmission load $\operatorname{Load}\left(v_{0}\right)=|\{2,3,5,8\}-\{2\}|=3$.

In practice, idle listening mode has nearly the same energy consumption rate with the receiving mode, which implies the receiving energy consumption for all nodes can be approximately neglected since in our model, each node only receives the packet at its scheduled time slot. In this paper, therefore, we will consider transmission load as the main metric of energy consumption.

In many broadcasting applications without cooperation requirement such as configuration dissemination, each node is expected to receive the broadcasting message to update the configuration as soon as possible. Thus, it is important to minimize the E2E delay of the broadcasting for such applications in lowduty-cycle WSNs. Here, we regard load balancing as the main consideration of energy efficiency, and in practice, the sink node is usually not energy-critical as it can be supplied with energy continuously, so it is unnecessary to consider the energy consumption of the sink node in our problem. Thus, our objective is to address the following Load-Balanced Minimum E2E-delay Broadcast Scheduling Problem (LB-MEBS) in low-duty-cycle WSNs. 
Table 2: Summary of the defined problems

\begin{tabular}{c|c|c|c}
\hline Abbreviation & Problem Full Name & Position & Hardness \\
\hline \hline LB-MEBS & $\begin{array}{c}\text { Load-Balanced Minimum } \\
\text { E2E-delay Broadcast } \\
\text { Scheduling Problem }\end{array}$ & $\begin{array}{c}\text { Problem 1, } \\
\text { Section 3.2 }\end{array}$ & NP-hard \\
\hline LBPA & $\begin{array}{c}\text { Load-Balanced Parents } \\
\text { Assignment Problem }\end{array}$ & $\begin{array}{c}\text { Problem 2, } \\
\text { Section 4 }\end{array}$ & NP-hard \\
\hline SLBS & $\begin{array}{c}\text { Spatiotemporal Load-Balanced } \\
\text { Semi-matching Problem }\end{array}$ & $\begin{array}{c}\text { Problem 3, } \\
\text { Section 4 }\end{array}$ & NP-hard \\
\hline DBS & $\begin{array}{c}\text { Degree-Balanced } \\
\text { Semi-matching Problem }\end{array}$ & $\begin{array}{c}\text { Problem 4, } \\
\text { Section 5.3 }\end{array}$ & P \\
\hline
\end{tabular}

Problem 1 (LB-MEBS). Given a spatiotemporal topology graph $G=(V, E$, $W, L)$, find a minimum E2E-delay spanning tree (i.e., Minimum Delay Path Tree (MDPT) where the E2E delay from the sink $v_{0}$ to each sensing node is minimal) $T^{*}$ rooted at the sink node $v_{0}$ so that the maximum transmission load of non-sink sensing nodes on $T^{*}$ is minimized.

In order to facilitate the reading, a summary of all the problems defined in this paper is given in Table 2 .

\subsection{Solution Overview}

In the following sections, we will prove the NP-hardness of our target LBMEBS problem and propose an approximation algorithm to solve this problem. Specifically, we first transform LB-MEBS problem into the equivalent LoadBalanced Parents Assignment Problem (LBPA) and show that it is NP-hard. Then, LBPA problem can further be transformed into several independent subproblems, and we can find that each subproblem is actually a generalization of the well-known Degree-Balanced Semi-matching Problem (DBS) and no existing solution was proposed for this problem. Based on the state-of-the-art solution to DBS problem, we propose an efficient greedy-like solution to solve each subproblem. Actually, the performance of our solution can be well-guaranteed as it benefits from the high efficiency of the state-of-the-art solution to DBS problem. Also, we come up with a fully distributed solution for our target problem. The basic idea is to let each node determine an initial transmission strategy set 
and then iteratively make a local competition at each node by exchanging a few of control packets between local nodes. If any node has the locally maximum transmission load at some round of the local competition, it will be the competition winner and drop one redundant transmission from its transmission strategy set, and this process will be repeated until there does not exist any redundant transmission for each node. Intuitively, our distributed solution would perform well as it adopts a locally greedy strategy to reduce the maximum transmission load in any local area. Finally, we will verify the high-efficiency of our proposed solutions by both theoretical analysis and extensive simulations.

\section{Problem Hardness Analysis}

In this section, we will analyze the hardness of our target problem. First, we construct a Minimum Delay Path Graph (MDPG) from $G$ which can make sure that the minimum E2E-delay constraint is satisfied. Then, we transform LBMEBS problem into the equivalent Load-Balanced Parents Assignment Problem (LBPA) on MDPG, which is independent of the minimum E2E delay constraint. Finally, we analyze the hardness of LBPA problem.

Here, we use $\operatorname{delay}\left(v_{0}, v_{i}\right)$ to denote the minimum E2E delay from sink $v_{0}$ to any node $v_{i}$ and we initially set $\operatorname{delay}\left(v_{0}, v_{0}\right)=0$, and $\operatorname{delay}\left(v_{0}, v_{i}\right)=\infty$ for any $v_{i} \neq v_{0}$. For any sensing node $v_{i}$, we use $C P S\left(v_{i}\right)$ and $C C S\left(v_{i}\right)$ to denote its Candidate Parents Set and Candidate Children Set respectively, where CPS $\left(v_{i}\right)$ and $C C S\left(v_{i}\right)$ are both initially set as null. For any node $v_{i}$, we define $T_{s}\left(v_{i}\right)=$ $\left\{t_{i}\right\}$. Further, we assume the sink node $v_{0}$ starts the broadcast operation at its scheduled time slot $t_{0}$ and we denote by $d_{i j}$ the point-to-point transmission delay for any edge $\left(v_{i}, v_{j}\right) \in E$, and $d_{i j}$ can be determined as follows:

If $v_{i}=v_{0}$,

$$
d_{i j}= \begin{cases}t_{j}-t_{i}+1, & \text { if } t_{j} \geq t_{i} \\ t_{j}-t_{i}+L+1, & \text { otherwise }\end{cases}
$$

and if $v_{i} \neq v_{0}$,

$$
d_{i j}= \begin{cases}t_{j}-t_{i}, & \text { if } t_{j}>t_{i} \\ t_{j}-t_{i}+L, & \text { otherwise }\end{cases}
$$


Next, we will first present the following Minimum Delay Path Graph Construction Algorithm (MDPGC-A), which is similar to Bellman-Ford Algorithm, to construct the Minimum Delay Path Graph (MDPG): Initially, each node will keep awake and determines its own working schedule depending on a particular power management protocol immediately after deployment, and then exchange the working schedule with its neighbors. Afterwards, the sink $v_{0}$ starts to broadcast a control packet containing its ID and $\operatorname{delay}\left(v_{0}, v_{0}\right)$. Upon receiving the broadcast control packet from any neighboring node $v_{i}$, sensing node $v_{j}$ will figure out $d_{i j}$ according to Equ. (2) and Equ. (3), and compares the current stored $\operatorname{delay}\left(v_{0}, v_{j}\right)$ with $\operatorname{delay}\left(v_{0}, v_{i}\right)+d_{i j}$.

- If $\operatorname{delay}\left(v_{0}, v_{i}\right)+d_{i j}<\operatorname{delay}\left(v_{0}, v_{j}\right)$, node $v_{j}$ will update the current $\operatorname{delay}\left(v_{0}, v_{j}\right)$ as $\operatorname{delay}\left(v_{0}, v_{i}\right)+d_{i j}$, then send a feedback packet to its neighbors to make node $v_{i}$ add $v_{j}$ into the set $C C S\left(v_{i}\right)$ and each node $v_{i}^{\prime} \in C P S\left(v_{j}\right)$ remove $v_{j}$ from the set $C C S\left(v_{i}^{\prime}\right)$ if $C P S\left(v_{j}\right) \neq \emptyset$, and next it will update $C P S\left(v_{j}\right)$ as $\left\{v_{i}\right\}$ and broadcast a new control packet containing its ID and the updated delay $\left(v_{0}, v_{j}\right)$;

- If $\operatorname{delay}\left(v_{0}, v_{i}\right)+d_{i j}=\operatorname{delay}\left(v_{0}, v_{j}\right)$, node $v_{j}$ will just add $v_{i}$ into the set $C P S\left(v_{j}\right)$ and reply a feedback packet to $v_{i}$ to make node $v_{i}$ add $v_{j}$ into the set $C C S\left(v_{i}\right)$;

- If $\operatorname{delay}\left(v_{0}, v_{i}\right)+d_{i j}>\operatorname{delay}\left(v_{0}, v_{j}\right)$, nothing is performed by node $v_{j}$.

The above process continues until no new control/feedback packet is received for each node.

Obviously, a MDPT can be obtained if any sensing node $v_{i}$ in MDPG selects any node in $C P S\left(v_{i}\right)$ as its parent. Our target problem, i.e., LB-MEBS problem, is thus equivalent to the following Load-Balanced Parents Assignment Problem (LBPA) which is independent of the minimum E2E delay constraint.

Problem 2 (LBPA). Given a spatiotemporal topology graph $G=(V, E, W, L)$ and its corresponding $M D P G$, for each non-sink sensing node $v_{i}$ in $M D P G$, choose a node in CPS $\left(v_{i}\right)$ to be its parent so that the maximum transmission load of non-sink sensing nodes on the resulting MDPT is minimized. 
Next, we will analyze the hardness of LBPA problem. First, we come up with the Spatiotemporal Load-Balanced Semi-matching Problem (SLBS) and prove that it is NP-hard. Then, we show the NP-hardness of LBPA problem by reduction from SLBS problem.

Definition 2 (Semi-matching). Given a spatiotemporal bipartite graph $G=$ $(S \cup R, E, W, L)$ where $S$ is the set of senders, $R$ is the set of receivers and $E \subseteq S \times R, W$ denotes the set of working schedules for all nodes in $S \cup R$, and $L$ denotes the schedule period length of each node, a semi-matching is defined as a set $M$ of edges with $M \subseteq E$ such that each node in $R$ is incident with exactly one edge in $M$.

Problem 3 (SLBS). Given a spatiotemporal bipartite graph $G=(S \bigcup R, E, W, L)$ where $S$ is the set of senders, $R$ is the set of receivers and $E \subseteq S \times R, W$ denotes the set of working schedules for all nodes in $S \bigcup R$, and $L$ denotes the schedule period length of each node, find a semi-matching $M$ in $G$ such that $\max _{s \in S}\left\{\operatorname{Load}_{M}(s)\right\}$ is minimized, where $\operatorname{Load}_{M}(s)$ denotes the transmission load of the sender $s$ in $M$.

Lemma 4.1. SLBS problem is NP-hard.

Proof. We refer to the decision version of SLBS problem as $D P_{S L B S}$ : Given a spatiotemporal bipartite graph $G=(S \bigcup R, E, W, L)$ and a non-negative integer $k$, is there a semi-matching so that the maximum transmission load of the senders in $S$ is at most $k$ ? We will prove that $D P_{S L B S}$ is NP-complete by reduction from the well-known NP-hard Monotone $3 S A T$ problem [28], where we are given a set $U$ of boolean variables and a collection $C$ of clauses over $U$ such that each clause $c \in C$ contains either only negated variables or only un-negated variables and $|c|=3$. The question is whether there is a satisfying truth assignment for $C$.

Given any instance of Monotone $3 S A T$ with variables $x_{1}, \ldots, x_{n}$ and clauses $c_{1}, \ldots, c_{m}$, construct the bipartite graph $G=(S \bigcup R, E, W, 3)$ where $S=$ $\left\{x_{1}, \ldots, x_{n}\right\}$ and $R=\left\{c_{1}, \ldots, c_{m}\right\}$, corresponding naturally to the set of variables and clauses. There is an edge $\left(x_{p}, c_{q}\right) \in E$ if and only if either $x_{p} \in c_{q}$ or $\bar{x}_{p} \in c_{q}$. The set $W$ is as follows. (1) $T_{s}(x)=\{0\}$ for all $x \in S$; (2) $T_{s}\left(c_{p}\right)=\{1\}$ if clause $c_{p}$ consists of only un-negated variables; (3) $T_{s}\left(c_{p}\right)=\{2\}$ if clause $c_{p}$ consists of only negated variables. The non-negative integer $k$ is set as 1 . It is easy to see that this reduction can be done in polynomial time. We give an example of the reduction. For the instance of the Monotone $3 S A T$ problem

$$
\left(x_{1} \vee x_{3} \vee x_{4}\right) \wedge\left(\bar{x}_{1} \vee \bar{x}_{2} \vee \bar{x}_{3}\right) \wedge\left(x_{2} \vee x_{4} \vee x_{5}\right) \wedge\left(\bar{x}_{2} \vee \bar{x}_{3} \vee \bar{x}_{5}\right)
$$

which consists of four clauses and $U=\left\{x_{1}, \ldots, x_{5}\right\}$, we obtain its corresponding instance of $D P_{S L B S}$ in Fig. 3.

We claim that the Monotone $3 S A T$ problem is satisfiable if and only if there exists a semi-matching in the constructed bipartite graph $G$ with a maximum transmission load of 1 . 
$\mathrm{R}$ :

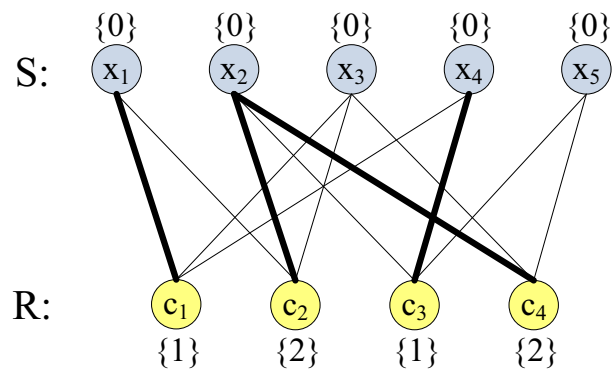

Figure 3: An instance of $D P_{S L B S}$ with $L=3$. Given any semi-matching with the maximum transmission load of 1 , e.g., the set of bold edges, we can easily get a truth assignment $t\left(\left\{x_{1}, x_{2}, x_{3}, x_{4}, x_{5}\right\}\right)=\{$ true, false, false, true, false $\}$.

i) Suppose an instance of Monotone $3 S A T$ problem is satisfiable and let $t: U \rightarrow\{$ true, false $\}$ be a satisfying truth assignment. We show that a desired semi-matching can be derived from this truth assignment. For node $x \in S$ such that $t(x)=$ true, we let it be parent of all neighbors whose active time slot is 1 , and for node $x \in S$ such that $t(x)=$ false, we let it be parent of all neighbors whose active time slot is 2 . In cases where a single node in $R$ is assigned multiple parents, we arbitrarily keep one of them and delete the others. Since each clause has at least one literal evaluating to true so that it has at least one parent, the edges between parents and children cover all nodes in $R$, forming a legitimate semi-matching. It is easy to see that this semi-matching must have a maximum transmission load of 1 .

ii) Suppose $M$ is a semi-matching for the constructed $D P_{S L B S}$ instance with a maximum transmission load of 1 . We can obtain a satisfying truth assignment $t$ for the Monotone $3 S A T$ problem. Due to maximum transmission load of 1, the children in $M$ of any node $x \in S$ have the same active time slot, either 1 or 2. Therefore, we let $t(x)=$ true if the active time slot of the children of node $x$ is 1 , and let $t(x)=$ false if either $x$ does not have child or its children have active time slot 2 . One can check that this truth assignment satisfies the Monotone $3 S A T$ problem.

It is obvious that $D P_{S L B S}$ is in NP since we can non-deterministically select a solution and check its transmission load in polynomial time. Therefore, $D P_{S L B S}$ is NP-complete, and the proof is completed.

Theorem 4.1. LBPA problem is NP-hard.

Proof. Given any instance $G=(S \bigcup R, E, W, L)$ of SLBS problem where $S=$ $\left\{s_{1}, \ldots, s_{n}\right\}, R=\left\{r_{1}, \ldots, r_{m}\right\}$ and suppose that $T_{s}(v)=\left\{t_{v}\right\}$ for any $v \in S \bigcup R$, we can construct an instance $G^{\prime}=\left(V^{\prime}, E^{\prime}, W^{\prime}, L+1\right)$ of LBPA problem in polynomial-time as follows. First, we let $V^{\prime}=\left\{v_{0}\right\} \bigcup V_{s} \bigcup V_{r}$ where $V_{s}=$ $\left\{s_{1}, \ldots, s_{n}\right\}, V_{r}=\left\{r_{1}, \ldots, r_{m}\right\}$ and $v_{0}$ denotes the sink node, and $E^{\prime}=\left(\left\{v_{0}\right\} \times\right.$ $\left.V_{s}\right) \cup E$. Then, for any node $r_{q} \in V_{r}$, if there exists at least one node $s_{p}$ in $V_{s}$ so that $T_{s}\left(s_{p}\right)=T_{s}\left(r_{q}\right)$, we will update $V_{r}$ by removing node $r_{q}$ from $V_{r}$, and 
update $E^{\prime}$ by removing all the edges that are attached to $r_{q}$ from $E^{\prime}$. Next, we set $W^{\prime}$ by letting 1$) T_{s}\left(v_{0}\right)=\{0\}$;2) $T_{s}\left(s_{p}\right)=\{0\}$ for any node $s_{p} \in V_{s} ; 3$ ) $T_{s}\left(r_{q}\right)=\left\{t_{r_{q}}+1\right\}$ for any node $r_{q} \in V_{r}$.

By such a construction, we can easily prove that the optimal spatiotemporal load-balanced semi-matching on $G$ can be found in polynomial-time if and only if the optimal MDPT, in which the maximum transmission load of non-sink sensing nodes is minimized, can be found from $G^{\prime}$ in polynomial-time. As this proof is simple and obvious, we omit the detailed process for saving space. Accordingly, SLBS problem is polynomial-time reducible to LBPA problem. According to Lemma 4.1, the proof is now completed.

\section{Centralized Solution}

To solve our target problem, in this section, we will propose an efficient solution, i.e., Load-Balanced Parents Assignment Algorithm (LBPA-A), which mainly consists of (1) Component Construction; (2) Component Simplification; (3) Degree-balanced Semi-matching; (4) Redundant Transmissions Reduction.

\subsection{Component Construction}

Definition 3 (Component). Given a spatiotemporal topology graph $G=(V, E$, $W, L)$ and its $M D P G$, we can define an undirected bipartite graph $G_{c}=\left(S_{c} \cup R_{c}\right.$, $\left.E_{c}, W, L\right)$ to characterize the minimum E2E-delay broadcasting in low-dutycycle WSNs, where $S_{c}=V$ is the set of potential senders, $R_{c}=V$ is the set of potential receivers and $E_{c} \subseteq S_{c} \times R_{c}$. For any $s \in S_{c}$ and any $r \in R_{c}$, specifically, $E_{c}$ contains an edge that connecting $s$ and $r$ if and only if $s \in C P S(r)$. In this paper, a component is defined as a maximal connected bipartite subgraph of the bipartite graph $G_{c}$. Here, a subgraph of $G_{c}$ is called maximal connected if and only if it is connected and any other edge in $E_{c}$ that does not belong to this subgraph is not incident with it.

Based on the information about candidate parents for all nodes, we can easily construct the bipartite graph $G_{c}$ according to its definition mentioned above, and then derive all the components from $G_{c}$ by the following steps:

- Initially, we mark all the edges in $E_{c}$ with unselected.

- We assume that a component $\mathcal{C}$ initially only contains one edge that is arbitrarily selected from the unselected edges in $E_{c}$, and then we extend the component $\mathcal{C}$ by iteratively merging $\mathcal{C}$ with the edges incident to $\mathcal{C}$, until no edge incident to $\mathcal{C}$ is found in $E_{c}$. Next, we mark all the edges in the resulting $\mathcal{C}$ with selected. 

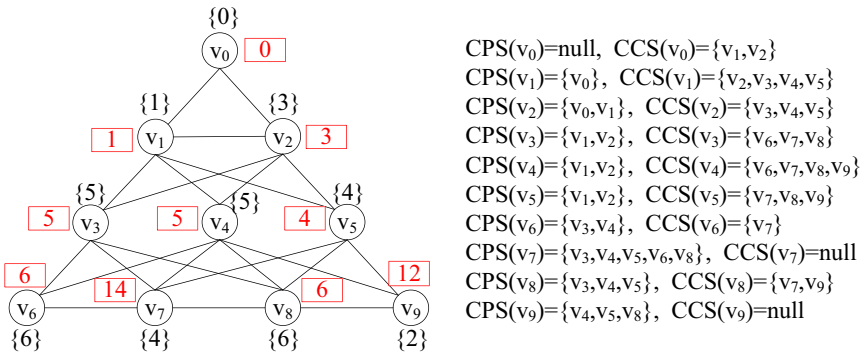

(a) The network topology graph $G$ with $L=10$

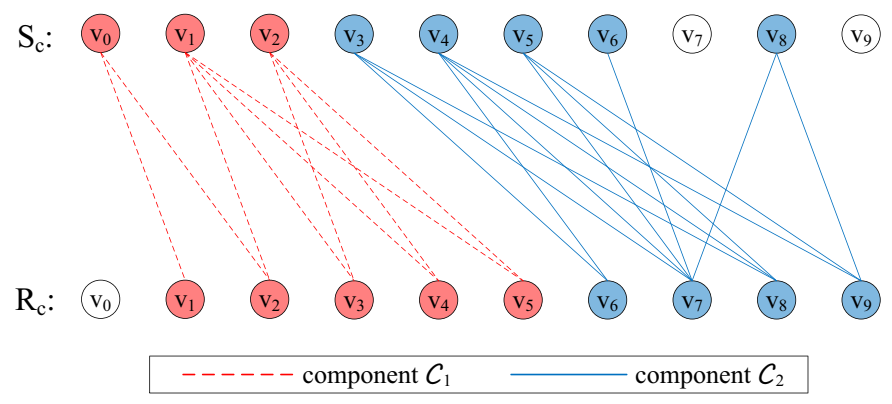

(b) The bipartite graph $G_{c}$

Figure 4: An example of component construction

- We repeat Step 2 until all the edges in $E_{c}$ are marked with selected, and finally all the components $\left\{\mathcal{C}_{1}, \mathcal{C}_{2}, \ldots, \mathcal{C}_{m}\right\}$ in $G_{c}$ can be found.

Fig. 4 explicitly illustrates an example of component construction. Given a network topology graph $G$ with $L=10$ as shown in Fig. 4(a), we can figure out the minimum E2E delay from the sink to each node in $G$, and obtain the Candidate Parents Sets for all nodes according to the aforementioned MDPG construction algorithm. As shown in Fig. 4(b), accordingly, we can easily construct the corresponding bipartite graph $G_{c}$ which consists of two components $\left\{\mathcal{C}_{1}, \mathcal{C}_{2}\right\}$

Observation 1. Components are independent of each other, that is, the parents assignment in one component will NOT affect that in any other component in terms of both delay-optimality and energy-fairness for all nodes.

According to Observation 1, it is obvious that LBPA problem can thus be transformed into several independent subproblems, i.e., SLBS problem on each 
component. Specifically, our objective is thus to solve the following independent subproblem: for any component $\mathcal{C}_{i}=\left(S_{i} \bigcup R_{i}, E_{i}, W_{i}, L\right)$ where $S_{i} \subseteq S_{c}, R_{i} \subseteq$ $R_{c}, E_{i} \subseteq E_{c}$ and $W_{i} \subseteq W$, find a semi-matching, namely assign exactly one parent from the senders in $S_{i}$ to each receiver in $R_{i}$, so that the maximum transmission load of the senders in $S_{i}$ is minimized.

\subsection{Component Simplification}

As stated before, we do not consider the energy consumption of the sink node in our problem due to the fact that the sink node can be always supplied with energy continuously in practice. For any neighbor of the sink node, we thus select the sink node as its parent. Correspondingly, we can simplify any component $\mathcal{C}_{i}=\left(S_{i} \cup R_{i}, E_{i}, W_{i}, L\right)$ by deleting all the edges that are incident to the nodes in $N_{0} \bigcap R_{i}$ from $\mathcal{C}_{i}$, where $N_{0}$ denotes the set of the sink node's neighbors. As shown in Fig. 4, the sink $v_{0}$ has two neighbors $\left\{v_{1}, v_{2}\right\}$, we thus let $v_{0}$ be the parent of both $v_{1}$ and $v_{2}$, and remove the edges $\left(v_{0}, v_{1}\right),\left(v_{0}, v_{2}\right)$ and $\left(v_{1}, v_{2}\right)$ from the component $\mathcal{C}_{1}$ to get a simplified component of $\mathcal{C}_{1}$.

Due to our power consumption assumption, actually, the transmission load of any sender $s_{i}^{p}$ will not involve the transmission from $s_{i}^{p}$ to any of its receivers that have the same working schedule with $s_{i}^{p}$. Therefore, we can further simplify any component $\mathcal{C}_{i}=\left(S_{i} \cup R_{i}, E_{i}, W_{i}, L\right)$ as follows: For any receiver $r_{i}^{q} \in R_{i}$, we check whether there exist one or multiple senders in $C P S\left(r_{i}^{q}\right)$ which have the same working schedule with $r_{i}^{q}$. If yes, we will let any one of them be the parent of $r_{i}^{q}$ and then delete all the edges that are incident to $r_{i}^{q}$ from $\mathcal{C}_{i}$. For example, node $v_{7}$ in Fig. 4(b) has one sender $v_{5}$ in $C P S\left(v_{7}\right)$ which has the same working schedule of $v_{7}$, i.e., $T_{s}\left(v_{5}\right)=T_{s}\left(v_{7}\right)=\{4\}$. Thus, we can assign $v_{5}$ as the parent of $v_{7}$ and remove the edges $\left(v_{3}, v_{7}\right),\left(v_{4}, v_{7}\right),\left(v_{5}, v_{7}\right),\left(v_{6}, v_{7}\right)$ and $\left(v_{8}, v_{7}\right)$ from the component $\mathcal{C}_{2}$ to get a simplified component of $\mathcal{C}_{2}$.

Finally, we update $C C S\left(s_{i}^{p}\right)$ for each sender $s_{i}^{p}$ in each simplified component $\mathcal{C}_{i}$, and by the above simplification, our objective will accordingly turn to be how to solve the SLBS problem on each simplified component. 


\subsection{Degree-balanced Semi-matching}

Before solving the SLBS problem, we first review the well-known DegreeBalanced Semi-matching Problem (DBS), which has been well-studied by many existing works $[29,30]$.

Problem 4 (DBS [29]). Given a bipartite graph $G=(S \cup R, E)$ where $S$ is the set of senders, $R$ is the set of receivers and $E \subseteq S \times R$, find a semi-matching $M$ in $G$ such that $\max _{s \in S}\left\{\operatorname{deg}_{M}(s)\right\}$ is minimized, where $\operatorname{deg}_{M}(s)$ denotes the degree of the sender $s$ in $M$, i.e., the number of edges in $M$ that are incident with $s$.

Obviously, our proposed SLBS problem is a generalization of the DBS problem. Specially, if the duty cycle in the network is so low that all the neighbors of any node have different working schedules, then $\operatorname{Load}_{M}(s)$ must be equal to $\operatorname{deg}_{M}(s)$ for any semi-matching $M$ and thus the SLBS problem will turn to be the DBS problem. As we know, it has been shown that the DBS problem is equivalent to the optimal semi-matching problem which is solvable in polynomial-time [29], and the state-of-the-art solution [30] of the DBS problem provides an efficient algorithm which runs in $O(\sqrt{|S \bigcup R|} \cdot|E| \cdot \log |S \bigcup R|)$ time. For convenience of description, we call the solution proposed in [30] the Degree-Balanced Semi-matching Algorithm (DBS-A).

Here, we will address the SLBS problem on any simplified component $\mathcal{C}_{i}$ by directly performing DBS-A on $\mathcal{C}_{i}$ and analyze its performance. Let $M_{i}^{d}$ denote the solution (i.e., the resulting semi-matching) when performing DBS-A on the simplified component $\mathcal{C}_{i}$, and $O P T\left(\mathcal{C}_{i}\right)$ denote the maximum transmission load of senders in the optimal solution of our target problem, i.e., the SLBS problem on $\mathcal{C}_{i}$. Also, we denote by $S\left(s_{i}^{p}, t\right)$ the set of message receivers in $C C S\left(s_{i}^{p}\right)$ if the sender $s_{i}^{p}$ transmits a message at time slot $t$ where $t \in \bigcup_{j \in C C S\left(s_{i}^{p}\right)} T_{s}(j)$, namely

$$
S\left(s_{i}^{p}, t\right)=\left\{r \mid r \in C C S\left(s_{i}^{p}\right) \text { and } T_{s}(r)=\{t\}\right\} .
$$

We have the following conclusion.

Lemma 5.1. Given any simplified component $\mathcal{C}_{i}=\left(S_{i} \bigcup R_{i}, E_{i}, W_{i}, L\right)$, we have

$$
\max _{s \in S_{i}}\left\{\operatorname{Load}_{M_{i}^{d}}(s)\right\} \leq \lambda_{i} \cdot O P T\left(\mathcal{C}_{i}\right)
$$


where $\lambda_{i}$ denotes the maximum number of the receivers that are scheduled to wake up at the same time and have at least one common candidate parent in $\mathcal{C}_{i}$,

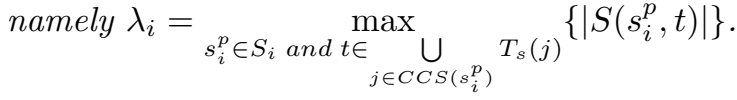

Proof. Let the semi-matching $M_{i}^{l}$ denote the optimal solution of the SLBS problem on $\mathcal{C}_{i}$ and define $s_{d}^{\max } \in \arg \max _{s \in S_{i}}\left\{\operatorname{deg}_{M_{i}^{l}}(s)\right\}$. We can obviously find that

$$
\begin{aligned}
\operatorname{OPT}\left(\mathcal{C}_{i}\right) & =\max _{s \in S_{i}}\left\{\operatorname{Load}_{M_{i}^{l}}(s)\right\} \\
& \geq \operatorname{Load}_{\mathrm{M}_{\mathrm{i}}^{1}}\left(\mathrm{~s}_{\mathrm{d}}^{\max }\right)
\end{aligned}
$$

and thus

$$
\begin{aligned}
\lambda_{i} \cdot O P T\left(\mathcal{C}_{i}\right) & \geq \lambda_{i} \cdot \operatorname{Load}_{M_{i}^{l}}\left(s_{d}^{\max }\right) \\
& \geq \operatorname{deg}_{\mathrm{M}_{\mathrm{i}}^{1}}\left(\mathrm{~s}_{\mathrm{d}}^{\max }\right) \\
& =\max _{\mathrm{s} \in \mathrm{S}_{\mathrm{i}}}\left\{\operatorname{deg}_{\mathrm{M}_{\mathrm{i}}^{1}}(\mathrm{~s})\right\} .
\end{aligned}
$$

Since $M_{i}^{d}$ is the semi-matching where the maximum degree of the senders in $\mathcal{C}_{i}$ is minimized, then

$$
\max _{s \in S_{i}}\left\{\operatorname{deg}_{M_{i}^{d}}(s)\right\} \leq \max _{s \in S_{i}}\left\{\operatorname{deg}_{M_{i}^{l}}(s)\right\} .
$$

Also, due to the fact that $\operatorname{Load}_{M_{i}^{d}}(s) \leq \operatorname{deg}_{M_{i}^{d}}(s)$ for any $s \in S_{i}$, we further define $s_{l}^{\max } \in \arg \max _{s \in S_{i}}\left\{\operatorname{Load}_{M_{i}^{d}}(s)\right\}$ and must have

$$
\begin{aligned}
\max _{s \in S_{i}}\left\{\operatorname{Load}_{M_{i}^{d}}(s)\right\} & =\operatorname{Load}_{M_{i}^{d}}\left(s_{l}^{\max }\right) \\
& \leq \operatorname{deg}_{\mathrm{M}_{\mathrm{i}}^{\mathrm{d}}}\left(\mathrm{s}_{1}^{\max }\right) \\
& \leq \max _{\mathrm{s} \in \mathrm{S}_{\mathrm{i}}}\left\{\operatorname{deg}_{\mathrm{M}_{\mathrm{i}}^{\mathrm{d}}}(\mathrm{s})\right\} .
\end{aligned}
$$

According to Equ. (6), (7) and (8), we can derive that

$$
\max _{s \in S_{i}}\left\{\operatorname{Load}_{M_{i}^{d}}(s)\right\} \leq \lambda_{i} \cdot \operatorname{OPT}\left(\mathcal{C}_{i}\right) .
$$

Thus, the proof is completed.

Theorem 5.1. For the LBPA problem, our solution that applies DBS-A to each simplified component achieves $\lambda$-approximation, where $\lambda$ denotes the maximum number of neighbors that are scheduled to wake up at the same time.

Proof. Suppose there are $m$ independent simplified components $\left\{\mathcal{C}_{1}, \ldots, \mathcal{C}_{m}\right\}$ in the problem. Let the found schedules by DBS-A be $M_{1}, \ldots, M_{m}$ respectively, and let the corresponding optimal schedules be $M_{1}^{*}, \ldots, M_{m}^{*}$. Due to Lemma 5.1 , for all $i$, we have

$$
\operatorname{Load}\left(M_{i}\right) \leq \lambda_{i} \operatorname{Load}\left(M_{i}^{*}\right)
$$


where $\operatorname{Load}(M)$ is the maximum load of all senders under schedule $M$. It is then straightforward to see that

$$
\max _{i} \operatorname{Load}\left(M_{i}\right) \leq \lambda \max _{i} \operatorname{Load}\left(M_{i}^{*}\right)
$$

since $\lambda \geq \lambda_{i}$ for all $i$. Note that the term $\max \operatorname{Load}\left(M_{i}\right)$ is the load of our solution and the term $\max _{i} \operatorname{Load}\left(M_{i}^{*}\right)$ is the load of the optimal solution.

In low-duty-cycle WSNs, we usually improve the network performance (e.g., to minimize average detection delay) by carefully designing the working schedules of all nodes (e.g., [23]) to make the neighboring nodes rotate the sensory coverage, which implies very few of the neighboring nodes will have the same working schedule in practice. Moreover, for a specific network performance requirement, it is usual that each node will correspondingly adjust (e.g., decrease) its duty cycle as the number of nodes $N$ varies (e.g., increase), which means $\lambda$ is actually independent of $N$ and only depends on the given network performance requirement. Thus, $\lambda$ is typically a small number in low-duty-cycle WSNs.

\subsection{Redundant Transmissions Reduction}

As shown in Theorem 5.1, the DBS-A can actually provide the performance guarantee in the worst case. However, we can find that some redundant transmissions would exist in the solution that adopting DBS-A. As a simple example, Fig. 5(a) shows a simplified component $\mathcal{C}_{i}$, and suppose that the set of the bold edges is the solution that adopting DBS-A on $\mathcal{C}_{i}$. Obviously, the transmission

from $s_{i}^{1}$ to $r_{i}^{1}$ is redundant since the receiver $r_{i}^{1}$ can also be covered by the transmission from $s_{i}^{2}$ to $r_{i}^{3}$. Likewise, the transmission from $s_{i}^{2}$ to $r_{i}^{4}$ is redundant since the receiver $r_{i}^{4}$ can also be covered by the transmission from $s_{i}^{3}$ to $r_{i}^{5}$. Thus, we can further make an improvement by removing the redundant edges $\left(s_{i}^{1}, r_{i}^{1}\right)$ and $\left(s_{i}^{2}, r_{i}^{4}\right)$ from our solution, which will reduce the maximum transmission load and the total transmission load from 2 and 6 to 1 and 4 respectively. In other words, the average case performance of our solution could be further improved by efficiently reducing the redundancy of transmissions. In this subsection, accordingly, we will devise an efficient heuristic approach to reduce the redundant transmissions in the solution adopting DBS-A. By doing 

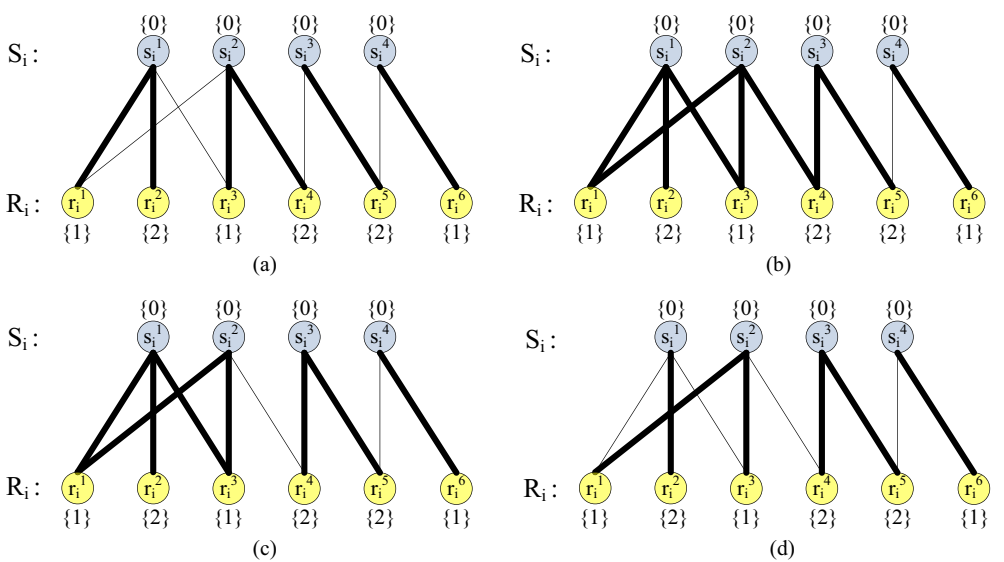

Figure 5: An example of redundant transmissions reduction

so, the performance of our solution can be further improved in terms of both energy-fairness and total energy consumption.

In order to better characterize the redundancy of transmissions, we utilize the Transmission Coverage Graph (TCG) to represent our solution (i.e., the semi-matching $M_{i}^{d}$ ) that adopting DBS-A on any simplified component $\mathcal{C}_{i}$, and $M_{i}^{d}$ can be easily transformed into its corresponding TCG, say $T_{i}^{d}$, as follows. Initially, $T_{i}^{d}$ consists of all the edges in $M_{i}^{d}$, and then any edge $\left(s_{i}^{p}, r\right) \notin M_{i}^{d}$ in $\mathcal{C}_{i}$ will be added into $T_{i}^{d}$ if and only if there exists at least one edge $\left(s_{i}^{p}, r_{i}^{q}\right)$ in $M_{i}^{d}$ where $T_{s}\left(r_{i}^{q}\right)=T_{s}(r)$. Obviously, our solution $M_{i}^{d}$ is equivalent to $T_{i}^{d}$ since in $M_{i}^{d}$, any transmission from $s_{i}^{p}$ to $r_{i}^{q}$ will also cover all the nodes with working schedule $T_{s}\left(r_{i}^{q}\right)$ in $C C S\left(s_{i}^{p}\right)$ due to the spatiotemporal nature of broadcasting. For the solution which consists of the bold edges in Fig. 5(a), we thus use the equivalent TCG which consists of the bold edges in Fig. 5(b) to represent it.

Here, let $\operatorname{Load}_{T_{i}^{d}}\left(s_{i}^{p}\right)$ denote the transmission load of the sender $s_{i}^{p}$ in $T_{i}^{d}$, $\operatorname{deg}_{T_{i}^{d}}\left(r_{i}^{q}\right)$ denote the degree of the receiver $r_{i}^{q}$ in $T_{i}^{d}$, and $C C S_{T_{i}^{d}}\left(s_{i}^{p}\right)$ denote the Candidate Children Set of the sender $s_{i}^{p}$ in $T_{i}^{d}$, i.e., $C C S_{T_{i}^{d}}\left(s_{i}^{p}\right)=\{r \mid r \in$ $C C S\left(s_{i}^{p}\right)$ and $\left.\left.\left(s_{i}^{p}, r\right) \in T_{i}^{d}\right)\right\}$. Also, we let $T R\left(s_{i}^{p}, t\right)\left(t \in \underset{j \in C C S_{T_{i}^{d}}\left(s_{i}^{p}\right)}{\bigcup} T_{s}(j)\right)$ denote one transmission of the sender $s_{i}^{p}$ at time slot $t$, which will cover all the nodes in $S\left(s_{i}^{p}, t\right)$, and $E_{i}\left(s_{i}^{p}, t\right)$ denote the set of edges that represents the 
transmission $T R\left(s_{i}^{p}, t\right)$, i.e., $E_{i}\left(s_{i}^{p}, t\right)=\left\{\left(s_{i}^{p}, r\right) \mid r \in S\left(s_{i}^{p}, t\right)\right\}$. For any transmission $T R\left(s_{i}^{p}, t\right)$ in our solution $T_{i}^{d}$, we will use the Redundancy Degree (RD) to characterize its redundancy level, which is defined as follows:

Definition 4 (Redundancy Degree (RD)). Given any simplified component $\mathcal{C}_{i}=\left(S_{i} \bigcup R_{i}, E_{i}, W_{i}, L\right)$ and our solution $T_{i}^{d}$, the Redundancy Degree (RD) of any transmission $T R\left(s_{i}^{p}, t\right)$ in $T_{i}^{d}$, saying $R D_{T_{i}^{d}}\left(s_{i}^{p}, t\right)$, is defined as

$$
R D_{T_{i}^{d}}\left(s_{i}^{p}, t\right)=\min _{r_{i}^{q} \in S\left(s_{i}^{p}, t\right)}\left\{\operatorname{deg}_{T_{i}^{d}}\left(r_{i}^{q}\right)\right\}
$$

where $s_{i}^{p} \in S_{i}$ and $t \in \underset{j \in C C S_{T_{i}^{d}}\left(s_{i}^{p}\right)}{ } T_{s}(j)$.

For any transmission $\operatorname{TR}\left(s_{i}^{p}, t\right)$ in $T_{i}^{d}$, it is called a redundant transmission if and only if $R D_{T_{i}^{d}}\left(s_{i}^{p}, t\right)>1$. If $R D_{T_{i}^{d}}\left(s_{i}^{p}, t\right)=1$, specially, it is called a necessary transmission since there must exist at least one receiver that can only be covered by the transmission $T R\left(s_{i}^{p}, t\right)$ in $T_{i}^{d}$.

In order to further improve the energy-fairness of our solution $T_{i}^{d}$ for any simplified component $\mathcal{C}_{i}$, we will propose a greedy algorithm, which consists of the following two steps, to efficiently reduce the redundant transmissions in $T_{i}^{d}$ :

Transmission Removal Step: First, we find out $S_{T_{i}^{d}}^{\max }$ which denotes the set of senders with the maximum transmission load in $T_{i}^{d}$, i.e., $S_{T_{i}^{d}}^{\max }=\arg \max _{s_{i}^{p} \in S_{i}}$ $\left\{\operatorname{Load}_{T_{i}^{d}}\left(s_{i}^{p}\right)\right\}$. For each transmission $T R\left(s_{i}^{p}, t\right)$ where $s_{i}^{p} \in S_{T_{i}^{d}}^{\max }$ and $t \in$ $\bigcup_{j \in C C S_{T_{i}^{d}}\left(s_{i}^{p}\right)} T_{s}(j)$, if it is a redundant transmission, we will define a metric $\Delta\left(s_{i}^{p}, t\right)$ as follows:

$$
\Delta\left(s_{i}^{p}, t\right)=\sum_{s \in S_{i}-\left\{s_{i}^{p}\right\}} \text { and } \sum_{t \in{ }_{j \in C C S_{T_{i}^{d}}(s)} T_{s}(j)} \frac{I\left(s, s_{i}^{p}, t\right) \cdot \operatorname{Load}_{T_{i}^{d}}(s)}{R D_{T_{i}^{d}}(s, t)}
$$

where

$$
I\left(s, s_{i}^{p}, t\right)= \begin{cases}1, & \text { if } R D_{T_{i}^{d}}(s, t) \neq R D_{T_{i}^{d}-E_{i}\left(s_{i}^{p}, t\right)}(s, t) \\ 0, & \text { otherwise }\end{cases}
$$

From all the redundant transmissions of the senders in $S_{T_{i}^{d}}^{m a x}$, we will first choose the one with the smallest $\Delta\left(s_{i}^{p}, t\right)^{1}$, say $T R\left(s^{*}, t^{*}\right)$, and then update $T_{i}^{d}$ as

\footnotetext{
${ }^{1}$ Specially, if there exist multiple redundant transmissions with the smallest $\Delta\left(s_{i}^{p}, t\right)$, we arbitrarily choose one from them.
} 
$T_{i}^{d}-E_{i}\left(s^{*}, t^{*}\right)$. Next, we repeat this step until there is no redundant transmission for all the senders in $S_{T_{i}^{d}}^{\max }$.

Transmission Replacement Step: When there is no redundant transmission for all the senders in $S_{T_{i}^{d}}^{\max }$, we will check each necessary transmission $\operatorname{TR}\left(s_{i}^{p}, t\right)$ of the senders in $S_{T_{i}^{d}}^{\max }$ that whether all the nodes in the set $S_{\text {deg=1 }}\left(s_{i}^{p}, t\right)=\left\{r \mid r \in S\left(s_{i}^{p}, t\right)\right.$ and $\left.\operatorname{deg}_{T_{i}^{d}}(r)=1\right\}$ can be covered by the transmissions out of $T_{i}^{d}$ while improving the energy-fairness. Specifically, we let $E_{i}^{r}\left(s_{i}^{p}, t\right)=\bigcup_{c} E_{i}(s, t)$ where $c=\left\{s \in S_{i}-\left\{s_{i}^{p}\right\}, t \notin \underset{j \in C C S_{T_{i}^{d}}(s)}{\bigcup} T_{s}(j)\right.$ and $\left.\operatorname{Load}_{T_{i}^{d}}(s) \leq \operatorname{Load}_{T_{i}^{d}}\left(s_{i}^{p}\right)-2\right\}$, and $T R\left(s_{i}^{p}, t\right)$ is called the replaceable necessary transmission if and only if $S_{d e g=1}\left(s_{i}^{p}, t\right) \subseteq E_{i}^{r}\left(s_{i}^{p}, t\right)$. We check that whether there exists any replaceable necessary transmission for all the senders in $S_{T_{i}^{d}}^{\max }$. If no, the algorithm is terminated; otherwise, we will arbitrarily choose one from them, say $T R\left(s^{*}, t^{*}\right)$, then update $T_{i}^{d}$ as $\left(T_{i}^{d}-E_{i}\left(s^{*}, t^{*}\right)\right) \cup E_{i}^{r}\left(s^{*}, t^{*}\right)$ and go back to the Transmission Removal Step.

In general, the basic idea of our greedy algorithm mentioned above is to greedily reduce the maximum transmission load in $T_{i}^{d}$ by each time either removing one redundant transmission of the sender with the maximum transmission load, or replacing one of its necessary transmissions with the out-of- $T_{i}^{d}$ transmissions from the other senders so that $\max _{s \in S_{i}}\left\{\operatorname{Load}_{T_{i}^{d}}(s)\right\}$ or $\left|S_{T_{i}^{d}}^{\max }\right|$ is reduced by 1 . For the sender with the maximum transmission load, the removal of one redundant transmission could influence (i.e., decrease) the RD values of the transmissions from the other senders, and when the $\mathrm{RD}$ value of one transmission decreases to 1 , it will be no longer redundant. Here, we employ the comparison metric $\Delta\left(s_{i}^{p}, t\right)$ to characterize the influence of the removal of the redundant transmission $T R\left(s_{i}^{p}, t\right)$ on the $\mathrm{RD}$ values of the transmissions from the other senders, which is related to three factors: the number of influenced transmissions that characterized by $I\left(s, s_{i}^{p}, t\right)$, the transmission load of any influenced transmission $T R(s, t)$ (i.e., $\operatorname{Load}_{T_{i}^{d}}(s)$ ) and the RD value of any influenced transmission $T R(s, t)$ (i.e., $R D_{T_{i}^{d}}(s, t)$ ). In order to achieve better energy-fairness, intuitively, we prefer to each time remove the one which will 
bring smaller number of influenced transmissions, smaller $\operatorname{Load}_{T_{i}^{d}}(s)$ and larger $R D_{T_{i}^{d}}(s, t)$ among all the redundant transmissions of the senders in $S_{T_{i}^{d}}^{\max }$.

Fig. 5 shows a simple example of our greedy algorithm on a simplified component $\mathcal{C}_{i}$, where the set of bold edges in Fig. 5(a) and that in Fig. 5(b) represent $M_{i}^{d}$ and $T_{i}^{d}$, respectively. In $T_{i}^{d}$, we can find that $S_{T_{i}^{d}}^{\max }=\left\{s_{i}^{1}, s_{i}^{2}\right\}$ and the transmission $T R\left(s_{i}^{2}, 2\right)$ has the smallest $\Delta\left(s_{i}^{p}, t\right)$ (i.e. $\Delta\left(s_{i}^{2}, 2\right)=0$ ) among all the redundant transmissions of the senders in $S_{T_{i}^{d}}^{\max }$. As shown in Fig. 5(c), thus, the redundant edge $\left(s_{i}^{2}, r_{i}^{4}\right)$ will be removed from $T_{i}^{d}$. Next, we can further find that $S_{T_{i}^{d}}^{\max }=\left\{s_{i}^{1}\right\}$, and as shown in Fig. 5(d), we will remove the redundant edges $\left(s_{i}^{1}, r_{i}^{1}\right)$ and $\left(s_{i}^{1}, r_{i}^{3}\right)$ from $T_{i}^{d}$ since the transmission $T R\left(s_{i}^{1}, 1\right)$ is currently the only redundant transmission of the senders in $T_{i}^{d}$. In Fig. $5(\mathrm{~d})$, it can be seen that the maximum transmission load has been decreased from 2 to 1 and $S_{T_{i}^{d}}^{\max }=\left\{s_{i}^{1}, s_{i}^{2}, s_{i}^{3}, s_{i}^{4}\right\}$, our greedy algorithm will thus be terminated since neither any redundant transmission nor any replaceable necessary transmission can be found among all the transmissions of the senders in $S_{T_{i}^{d}}^{\max }$. Intuitively, the greedy strategy with the comparison metric $\Delta\left(s_{i}^{p}, t\right)$ could have a better performance than that with the comparison metric $R D_{T_{i}^{d}}\left(s_{i}^{p}, t\right)$, since the latter does not consider the influence of the removed redundant transmission on any other redundant transmission in $T_{i}^{d}$. In this example, if we utilize the greedy strategy that each time removes the transmission with the largest $R D_{T_{i}^{d}}\left(s_{i}^{p}, t\right)$, instead of the transmission with the smallest $\Delta\left(s_{i}^{p}, t\right)$, from $T_{i}^{d}$ among all the redundant transmissions of the senders in $S_{T_{i}^{d}}^{\max }$, the transmission $T R\left(s_{i}^{2}, 1\right)$ (i.e., the redundant edges $\left(s_{i}^{2}, r_{i}^{1}\right)$ and $\left.\left(s_{i}^{2}, r_{i}^{3}\right)\right)$ would be first removed from $T_{i}^{d}$ which would cause the termination of the greedy algorithm without decreasing the maximum transmission load. This is because the removal of the transmission $\operatorname{TR}\left(s_{i}^{2}, 1\right)$ would affect the transmission $T R\left(s_{i}^{1}, 1\right)$ and make it no longer redundant.

By greedily removing or replacing the transmission of the sender with the maximum transmission load, we improve the performance of our solution in terms of energy-fairness. However, we can find that there could still exist redundant transmissions for the senders without the maximum transmission load after running our greedy algorithm, which suggests that the performance of 
our solution could be further improved in terms of total energy consumption. Specifically, our problem can be described as follows. Given a time slot $t$ where $t \in \bigcup_{r \in R_{i}} T_{s}(r)$ and a set of senders $S_{i}(t)=\left\{s \mid s \in S_{i}\right.$ and $\left.t \in \bigcup_{j \in C C S_{T_{i}^{d}}(s)} T_{s}(j)\right\}$, how to select a minimum subset of $S_{i}(t)$, say $S_{i}^{*}(t)$, so that $\bigcup_{s \in S_{i}^{*}(t)} S(s, t)=$ $\left\{r \mid r \in R_{i}\right.$ and $\left.T_{s}(r)=\{t\}\right\}$. Obviously, this is a typical Set Cover Problem which is NP-hard and can be well-solved with the state-of-the-art solution. In our solution, finally, we will remove all the edges in $\bigcup_{s \in S_{i}(t)-S_{i}^{*}(t)} E_{i}(s, t)$ from $T_{i}^{d}$ for each $t \in \bigcup_{r \in R_{i}} T_{s}(r)$.

Accordingly, we can find that our solution adopting DBS-A on $\mathcal{C}_{i}$ can be further improved in terms of both energy-fairness and total energy consumption by the aforementioned approach.

\subsection{Improvement}

The SLBS problem has been shown as NP-hard by Lemma 4.1. However, we can find that it is solvable in polynomial-time for some special instances of the simplified component, which indicates that the average performance of LBPA-A could be further improved by first checking whether each simplified component belongs to this kind of special instances. The following theorem illustrates the sufficient condition that the SLBS problem is solvable in polynomial-time.

Theorem 5.2. Given a simplified component $\mathcal{C}_{i}=\left(S_{i} \cup R_{i}, E_{i}, W_{i}, L\right)$, if $S\left(s_{i}^{p}, t\right)$ $\nsubseteq \bigcup_{s \in S_{i}-\left\{s_{i}^{p}\right\}} f(s, t)$ for each $s_{i}^{p} \in S_{i}$ and each $t \in \bigcup_{j \in C C S\left(s_{i}^{p}\right)} T_{s}(j)$, then there must exist an efficient algorithm so that the $S L B S$ problem on $\mathcal{C}_{i}$ can be solved in polynomial-time. Specifically,

$$
f(s, t)= \begin{cases}S(s, t), & \text { if } S(s, t) \neq S\left(s_{i}^{p}, t\right) \\ \text { null, }, & \text { otherwise }\end{cases}
$$

Proof. By capturing the spatiotemporal characteristic of broadcasting, we can make a component transformation for any simplified component $\mathcal{C}_{i}=\left(S_{i} \cup R_{i}\right.$, $\left.E_{i}, W_{i}, L\right)$ as follows. First, we define a bipartite graph $G^{T}=\left(S_{i} \cup V, E^{T}\right)$ where $V$ and $E^{T}$ are both initially set as null. For each $s_{i}^{p} \in S_{i}$ and each $t \in \bigcup_{j \in C C S\left(s_{i}^{p}\right)} T_{s}(j)$ in $\mathcal{C}_{i}$, we will check that whether there exists a vertex $v$ that represents the set $S\left(s_{i}^{p}, t\right)$ in $V$. If yes, we will add an edge $\left(s_{i}^{p}, v\right)$ into $E^{T}$; otherwise, we will add the vertex $v$ that represents the set $S\left(s_{i}^{p}, t\right)$ into $V$ and then add an edge $\left(s_{i}^{p}, v\right)$ into $E^{T}$. 

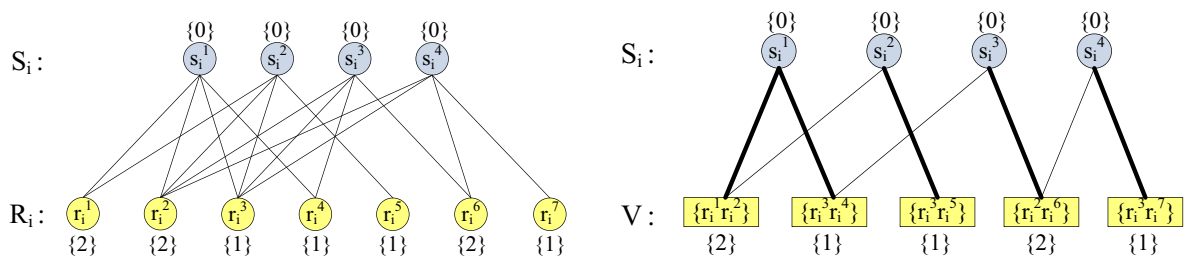

(a) The original simplified component $\mathcal{C}_{i}$ with $L=3$

(b) The transformed simplified component

Figure 6: An example of component transformation

Obviously, the above component transformation can be done in polynomialtime. Actually, the transformed simplified component $G^{T}$, in which one transmission is represented by just one edge, explicitly exhibits the spatiotemporal characteristic of broadcasting. For any sender $s_{i}^{p} \in S_{i}$ and any $t \in \bigcup_{j \in C C S\left(s_{i}^{p}\right)} T_{s}(j)$ in $\mathcal{C}_{i}, S\left(s_{i}^{p}, t\right) \nsubseteq \underset{s \in S_{i}-\left\{s_{i}^{p}\right\}}{\bigcup} f(s, t)$ implies that in the corresponding $G^{T}$, the vertex $S\left(s_{i}^{p}, t\right)$ in $V$ must contain at least one receiver which does not belong to any other vertex in $V$. Therefore, if $S\left(s_{i}^{p}, t\right) \nsubseteq \underset{s \in S_{i}-\left\{s_{i}^{p}\right\}}{\bigcup} f(s, t)$ for each $s_{i}^{p} \in S_{i}$ and each $t \in \bigcup_{j \in C C S\left(s_{i}^{p}\right)} T_{s}(j)$ in some $\mathcal{C}_{i}$, our target problem, i.e., how to find a SLBS in such a $\mathcal{C}_{i}$ where each receiver should be covered, can be equivalent to that how to find a DBS in its corresponding transformed simplified component $G^{T}$. Accordingly, we can solve the SLBS problem on such a $\mathcal{C}_{i}$ in polynomialtime by directly performing DBS-A on its corresponding transformed simplified component $G^{T}$.

Fig. 6 illustrates a simple example of the component transformation. For the simplified component $\mathcal{C}_{i}$ (i.e., Fig. 6(a)), we can find that it has satisfied the sufficient condition in Theorem 5.2. Therefore, we can solve the SLBS problem on this $\mathcal{C}_{i}$ in polynomial-time by adopting DBS-A on its corresponding transformed simplified component $G^{T}$ (i.e., Fig. 6(b)). According to the solution adopting DBS-A on $G_{T}$ which is represented by the set of bold edges in Fig. 6(b), we can get our final broadcasting schedule as $\left\{T R\left(s_{i}^{1}, 1\right), T R\left(s_{i}^{1}, 2\right), T R\left(s_{i}^{2}, 1\right), T R\left(s_{i}^{3}, 2\right), T R\left(s_{i}^{4}, 1\right)\right\}$. 


\section{Distributed Implementation}

Different from the aforementioned centralized solution which requires the global topology information, in this section, an efficient distributed approach, which we call the Distributed Load-Balanced Parents Assignment Algorithm (DLBPA-A), will be proposed to solve our target problem.

\subsection{Algorithm Description}

Definition 5 (Local Node). Given a spatiotemporal topology graph $G=$ $(V, E, W, L)$ and any two nodes $v_{i}, v_{j} \in V$, node $v_{i}$ is called the local node of node $v_{j}$ if and only if $C C S\left(v_{i}\right) \cap C C S\left(v_{j}\right) \neq \emptyset$ and there exists at least one node $v_{k} \in C C S\left(v_{i}\right) \cap C C S\left(v_{j}\right)$ so that $T_{s}\left(v_{k}\right) \neq T_{s}(r)$ for each $r \in C P S\left(v_{k}\right)$.

Here, we come up with an efficient distributed algorithm DLBPA-A, which mainly consists of Initialization Phase and Competition Phase.

Overall, in Initialization Phase, each node will figure out its initial transmission load and send it to all of its local nodes. In Competition Phase, each node will compete with its local nodes, and the competition winner which has the local maximum transmission load will make the redundancy reduction decision. This process will be repeated until no redundancy is found for each node.

Initialization Phase: First, we will perform the MDPGC-A as stated in Section 4 in a distributed way, accordingly, each node $v_{i}$ will get its $C P S\left(v_{i}\right)$ and $C C S\left(v_{i}\right)$, and the Transmission Strategy of each node $v_{i}$, say $T S\left(v_{i}\right)$, will be initially set as $\left\{T R\left(v_{i}, t\right) \mid t \in \bigcup_{j \in C C S\left(v_{i}\right)} T_{s}(j)\right\}$. If $C C S\left(v_{i}\right)=\emptyset$, in particular, $T S\left(v_{i}\right)$ will be initially set as null. Then, each sensing node $v_{i}$ will make the following decision:

- if $v_{0} \in C P S\left(v_{i}\right), v_{i}$ will choose $v_{0}$ as its parent, then mark itself with assigned node and send a control packet containing the assigned parent ID $v_{0}$ to all nodes in $C P S\left(v_{i}\right)$;

- if $v_{0} \notin C P S\left(v_{i}\right)$ and there exists a nonempty set $S^{\prime}=\left\{j \mid j \in C P S\left(v_{i}\right)\right.$ and $\left.T_{s}(j)=T_{s}\left(v_{i}\right)\right\}, v_{i}$ will arbitrarily choose one node in $S^{\prime}$ as its parent, then mark itself with assigned node and send a control packet containing the assigned parent ID to all nodes in $C P S\left(v_{i}\right)$; 
- if $v_{0} \notin C P S\left(v_{i}\right)$ and there does not exist a nonempty set $S^{\prime}=\{j \mid j \in$ $C P S\left(v_{i}\right)$ and $\left.T_{s}(j)=T_{s}\left(v_{i}\right)\right\}, v_{i}$ will mark itself with unassigned node, then set its degree $\operatorname{deg}\left(v_{i}\right)=\left|C P S\left(v_{i}\right)\right|$ and send a control packet containing $C P S\left(v_{i}\right)$ and $\operatorname{deg}\left(v_{i}\right)$ to all nodes in $C P S\left(v_{i}\right)$.

Afterwards, each node $v_{i}$ will wait until the control packets from all nodes in $C C S\left(v_{i}\right)$ are received. For each $t \in \bigcup_{j \in C C S\left(v_{i}\right)} T_{s}(j)$, if the nodes in $S\left(v_{i}, t\right)$ are all the assigned nodes and none of their assigned parent IDs is $v_{i}$, then $v_{i}$ will remove the transmission $T R\left(v_{i}, t\right)$ from $T S\left(v_{i}\right)$. Next, $v_{i}$ will update $C C S\left(v_{i}\right)$ by removing all the assigned nodes from $C C S\left(v_{i}\right)$. Based on the received control packets from all the unassigned nodes in $C C S\left(v_{i}\right), v_{i}$ will first figure out its transmission load $\operatorname{Load}\left(v_{i}\right)=\left|\bigcup_{j \in C C S\left(v_{i}\right)} T_{s}(j)\right|$ and find its local nodes set $L N S\left(v_{i}\right)=\bigcup_{j \in C C S\left(v_{i}\right)} C P S(j)-\left\{v_{i}\right\}$, and then send a competition packet containing $\operatorname{Load}\left(v_{i}\right)$ to all the nodes in $\operatorname{LNS}\left(v_{i}\right)$ by selecting the nodes in $C C S\left(v_{i}\right)$ as the forwarders. Specially, if $C C S\left(v_{i}\right)=\emptyset$, then $\operatorname{Load}\left(v_{i}\right)=0$ and no competition packet will be sent by $v_{i}$.

Competition Phase: Upon receiving the competition packets from all the nodes in $L N S\left(v_{i}\right), v_{i}$ will figure out its local maximum-load nodes set $L_{\max }\left(v_{i}\right)=$ $\arg \max _{j \in L N S\left(v_{i}\right) \cup\left\{v_{i}\right\}} \operatorname{Load}(j)$. If $v_{i}$ is the competition winner (i.e., $v_{i} \in L_{\text {max }}\left(v_{i}\right)$ and also $v_{i}$ has the smallest ID number among all the nodes in $\left.L_{\max }\left(v_{i}\right)\right)$, it will first figure out the Redundancy Degree (RD) $R D\left(v_{i}, t\right)=\min _{j \in S\left(v_{i}, t\right)} \operatorname{deg}(j)$ for each transmission $T R\left(v_{i}, t\right)\left(t \in \bigcup_{j \in C C S\left(v_{i}\right)} T_{s}(j)\right)$ and count the number of redundant transmissions $\left|T S_{r}\left(v_{i}\right)\right|$ where the redundant transmissions set $T S_{r}\left(v_{i}\right)=\left\{T R\left(v_{i}, t\right) \mid T R\left(v_{i}, t\right) \in T S\left(v_{i}\right)\right.$ and $t \notin T_{s}\left(v_{i}\right)$ and $\left.R D\left(v_{i}, t\right)>1\right\}$, and then make the following redundancy reduction decision:

- if $\left|T S_{r}\left(v_{i}\right)\right|>\operatorname{Load}\left(v_{i}\right)-\max _{j \in \operatorname{LNS}\left(v_{i}\right)} \operatorname{Load}(j)+1, v_{i}$ will first update $T S\left(v_{i}\right)=$ $T S\left(v_{i}\right)-T S_{r}^{*}\left(v_{i}\right)$ where $T S_{r}^{*}\left(v_{i}\right)$ denotes the set of redundant transmissions with the largest $\operatorname{Load}\left(v_{i}\right)-\max _{j \in \operatorname{LNS}\left(v_{i}\right)} \operatorname{Load}(j)+1 \mathrm{RD}$ values in $T S_{r}\left(v_{i}\right)$ and update $\operatorname{Load}\left(v_{i}\right)=\max _{j \in L N S\left(v_{i}\right)} \operatorname{Load}(j)-1$, then send a competition packet containing $\operatorname{Load}\left(v_{i}\right)$ and $T\left(v_{i}\right)$ to all the nodes in $C C S\left(v_{i}\right)$ where 
$T\left(v_{i}\right)=\left\{t \mid T R\left(v_{i}, t\right) \in T S_{r}^{*}\left(v_{i}\right)\right\} ;$

- if $\left|T S_{r}\left(v_{i}\right)\right| \leq \operatorname{Load}\left(v_{i}\right)-\max _{j \in \operatorname{LNS}\left(v_{i}\right)} \operatorname{Load}(j)+1, v_{i}$ will first update $T S\left(v_{i}\right)=$ $T S\left(v_{i}\right)-T S_{r}\left(v_{i}\right)$ and set $\operatorname{Load}\left(v_{i}\right)=0$, then send a competition packet containing $\operatorname{Load}\left(v_{i}\right)$ and $T\left(v_{i}\right)$ to all the nodes in $C C S\left(v_{i}\right)$ where $T\left(v_{i}\right)=$ $\left\{t \mid T R\left(v_{i}, t\right) \in T S_{r}\left(v_{i}\right)\right\}$.

Upon receiving this competition packet, any $v_{j} \in C C S\left(v_{i}\right)$ will 1) check whether $\operatorname{Load}\left(v_{i}\right)$ in this competition packet is 0 . If yes, it will add $v_{i}$ into a set $C P S_{0}\left(v_{j}\right)$ which is initially set as null; 2) check whether $T_{s}\left(v_{j}\right) \subseteq T\left(v_{i}\right)$. If no, $v_{j}$ will just forward a new competition packet containing $\operatorname{Load}\left(v_{i}\right)$ to all the nodes in $C P S\left(v_{j}\right)-C P S_{0}\left(v_{j}\right)$; if yes, $v_{j}$ will update $\operatorname{deg}\left(v_{j}\right)=\operatorname{deg}\left(v_{j}\right)-1$ and then forward a new competition packet containing $\operatorname{Load}\left(v_{i}\right)$ and $\operatorname{deg}\left(v_{j}\right)$ to all the nodes in $C P S\left(v_{j}\right)-C P S_{0}\left(v_{j}\right)$. For any node $v_{i}$ with non-zero transmission load, when receiving all the competition packets attached with the transmission load of any $v_{j} \in L N S\left(v_{i}\right)$ from all nodes in $C C S\left(v_{i}\right) \cap C C S\left(v_{j}\right)$, it will first update the current $\operatorname{Load}\left(v_{j}\right)$ and update $\operatorname{deg}(k)$ for each $k \in C C S\left(v_{i}\right) \cap C C S\left(v_{j}\right)$, then check whether it is the competition winner. If no, it will just keep waiting; if yes, it will recompute the $\mathrm{RD}$ value of each transmission, make the aforementioned redundancy reduction decision and then keep waiting. The above process is iteratively performed until each of the nodes becomes the terminated node and any node $v_{i}$ is called the terminated node if and only if either $\operatorname{Load}\left(v_{i}\right)=0$, or $\operatorname{Load}(j)=0$ for each $j \in \operatorname{LNS}\left(v_{i}\right)$.

Finally, any node $v_{i}$ will go into the low-duty-cycle mode according to the working schedule immediately after it becomes the terminated node, and once receiving a broadcasting message, $v_{i}$ will forward it according to the Transmission Strategy TS $\left(v_{i}\right)$.

\subsection{Example}

Fig. 7 illustrates an example of our proposed DLBPA-A on the component as shown in Fig. 7(a). Initially, each node in $S_{i}$ will set its Transmissions Set as follows (i.e., Fig. 7(b)):

$$
T S\left(s_{i}^{1}\right)=\left\{T R\left(s_{i}^{1}, 1\right), T R\left(s_{i}^{1}, 2\right)\right\} ;
$$



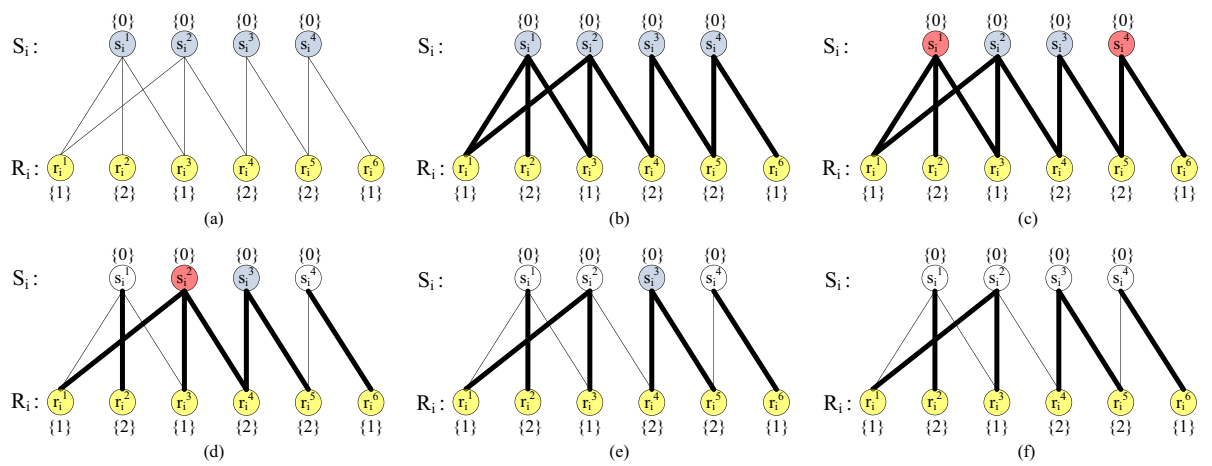

Figure 7: An example of distributed solution (red nodes denote competition winners and white nodes denote terminated nodes)

$$
\begin{gathered}
T S\left(s_{i}^{2}\right)=\left\{T R\left(s_{i}^{2}, 1\right), T R\left(s_{i}^{2}, 2\right)\right\} \\
T S\left(s_{i}^{3}\right)=\left\{T R\left(s_{i}^{3}, 2\right)\right\} ; \quad T S\left(s_{i}^{4}\right)=\left\{T R\left(s_{i}^{4}, 1\right), T R\left(s_{i}^{4}, 2\right)\right\} .
\end{gathered}
$$

then each node $r_{i}^{q} \in R_{i}$ will identify itself as the unassigned node and send a control packet containing $C P S\left(r_{i}^{q}\right)$ and $\operatorname{deg}\left(r_{i}^{q}\right)$ to all nodes in $C P S\left(r_{i}^{q}\right)$. Accordingly, each node in $S_{i}$ will respectively get its transmission load and local nodes set as follows:

$$
\begin{gathered}
\operatorname{Load}\left(s_{i}^{1}\right)=2, \quad \operatorname{LNS}\left(s_{i}^{1}\right)=\left\{s_{i}^{2}\right\} ; \\
\operatorname{Load}\left(s_{i}^{2}\right)=2, \quad \operatorname{LNS}\left(s_{i}^{2}\right)=\left\{s_{i}^{1}, s_{i}^{3}\right\} ; \\
\operatorname{Load}\left(s_{i}^{3}\right)=1, \quad \operatorname{LNS}\left(s_{i}^{3}\right)=\left\{s_{i}^{2}, s_{i}^{4}\right\} ; \\
\operatorname{Load}\left(s_{i}^{4}\right)=2, \quad \operatorname{LNS}\left(s_{i}^{4}\right)=\left\{s_{i}^{3}\right\} .
\end{gathered}
$$

Next, each node $s_{i}^{p} \in S_{i}$ will send a competition packet containing $\operatorname{Load}\left(s_{i}^{p}\right)$ to all the nodes in $\operatorname{LNS}\left(s_{i}^{p}\right)$, and thus $s_{i}^{1}$ will become the competition winner since $s_{i}^{1} \in L_{\max }\left(s_{i}^{1}\right)=\left\{s_{i}^{1}, s_{i}^{2}\right\}$ and $s_{i}^{1}$ has the smaller ID number than $s_{i}^{2}$. Likewise, $s_{i}^{4}$ will also become the competition winner since it has a larger transmission load than $s_{i}^{3}$ (i.e., Fig. $7(\mathrm{c})$ ).

Afterwards, $s_{i}^{1}$ and $s_{i}^{4}$ will respectively find that $T S_{r}\left(s_{i}^{1}\right)=\left\{T R\left(s_{i}^{1}, 1\right)\right\}$ and $T S_{r}\left(s_{i}^{4}\right)=\left\{T R\left(s_{i}^{4}, 2\right)\right\}$, and according to our redundancy reduction decision, $s_{i}^{1}$ will update $T S\left(s_{i}^{1}\right)=\left\{T R\left(s_{i}^{1}, 2\right)\right\}$, set $\operatorname{Load}\left(s_{i}^{1}\right)=0$ and send a competition 
packet containing $\operatorname{Load}\left(s_{i}^{1}\right)$ and $T\left(s_{i}^{1}\right)$ to all the nodes in $C C S\left(s_{i}^{1}\right)$. Similarly, $s_{i}^{4}$ will update $T S\left(s_{i}^{4}\right)=\left\{T R\left(s_{i}^{4}, 1\right)\right\}$, set $\operatorname{Load}\left(s_{i}^{4}\right)=0$ and send a competition packet containing $\operatorname{Load}\left(s_{i}^{4}\right)$ and $T\left(s_{i}^{4}\right)$ to all the nodes in $C C S\left(s_{i}^{4}\right)$, then $s_{i}^{1}$ and $s_{i}^{4}$ will both identify themselves as terminated nodes, and 1) $r_{i}^{1}$ will update $\operatorname{deg}\left(r_{i}^{1}\right)=1$ and then send a new competition packet containing $\operatorname{Load}\left(s_{i}^{1}\right)$ and $\operatorname{deg}\left(r_{i}^{1}\right)$ to $s_{i}^{2}$;2) $r_{i}^{3}$ will update $\operatorname{deg}\left(r_{i}^{3}\right)=1$ and then send a new competition packet containing $\operatorname{Load}\left(s_{i}^{1}\right)$ and $\operatorname{deg}\left(r_{i}^{3}\right)$ to $\left.s_{i}^{2} ; 3\right) r_{i}^{5}$ will update $\operatorname{deg}\left(r_{i}^{5}\right)=1$ and then send a new competition packet containing $\operatorname{Load}\left(s_{i}^{4}\right)$ and $\operatorname{deg}\left(r_{i}^{5}\right)$ to $s_{i}^{3}$. Next, $s_{i}^{2}$ will become the competition winner (i.e., Fig. $7(\mathrm{~d})$ ) and according to our redundancy reduction decision, $s_{i}^{2}$ will first update $\operatorname{TS}\left(s_{i}^{2}\right)=\left\{\operatorname{TR}\left(s_{i}^{2}, 1\right)\right\}$, set $\operatorname{Load}\left(s_{i}^{2}\right)=0$ and send a competition packet containing $\operatorname{Load}\left(s_{i}^{2}\right)$ and $T\left(s_{i}^{2}\right)$ to all the nodes in $C C S\left(s_{i}^{2}\right)$, and then identify itself as terminated node (i.e., Fig. $7(\mathrm{e}))$.

Finally, $r_{i}^{4}$ will update $\operatorname{deg}\left(r_{i}^{4}\right)=1$ and then send a new competition packet containing $\operatorname{Load}\left(s_{i}^{2}\right)$ and $\operatorname{deg}\left(r_{i}^{4}\right)$ to $s_{i}^{3}$, and $s_{i}^{3}$ will identify itself as terminated node since $\operatorname{Load}(j)=0$ for each $j \in \operatorname{LNS}\left(s_{i}^{3}\right)$, therefore, we can get the final broadcasting schedule (i.e., Fig. 7(f)) as follows:

$$
\begin{aligned}
& T S\left(s_{i}^{1}\right)=\left\{T R\left(s_{i}^{1}, 2\right)\right\} ; \quad T S\left(s_{i}^{2}\right)=\left\{T R\left(s_{i}^{2}, 1\right)\right\} ; \\
& T S\left(s_{i}^{3}\right)=\left\{T R\left(s_{i}^{3}, 2\right)\right\} ; \quad T S\left(s_{i}^{4}\right)=\left\{T R\left(s_{i}^{4}, 1\right)\right\} .
\end{aligned}
$$

\subsection{Message Complexity}

In Initialization Phase, it is obvious that the MDPGC-A will cost $O\left(N^{2}\right)$ transmissions where $N$ denotes the number of nodes in the network. After the MDPGC-A, each sensing node $v_{i}$ will send one control packet to all nodes in $C P S\left(v_{i}\right)$ and some of nodes will then respectively send one competition packet. Upon receiving a competition packet from any node in $C P S\left(v_{i}\right)$, any unassigned node $v_{i}$ will forward this competition packet to all nodes in $C P S\left(v_{i}\right)$. As we know, $\left|C P S\left(v_{i}\right)\right| \leq d_{\max }$ where $d_{\max }$ denotes the maximum node degree in the network. Let $C O S T_{\text {init }}$ denote the number of transmissions in Initialization Phase, and we can have that $C O S T_{\text {init }} \leq O\left(N^{2}\right)+N+N+N \cdot d_{\max }=O\left(N^{2}\right)$. 
In Competition Phase, once any node $v_{i}$ is determined as the competition winner, it will send one competition packet to all nodes in $C C S\left(v_{i}\right)$ and each node $v_{j} \in C C S\left(v_{i}\right)$ will then send at most one competition packet to all nodes in $C P S\left(v_{j}\right)-C P S_{0}\left(v_{j}\right)$. As $\left|C C S\left(v_{i}\right)\right| \leq d_{\max }$, the event that any node is determined as the competition winner will result in at most $1+d_{\max }$ transmissions. Let COST comp denote the number of transmissions in Competition Phase, and it is obvious that any node $v_{i}$ will determine itself as the competition winner for at most $\left|\bigcup_{j \in C C S\left(v_{i}\right)} T_{s}(j)\right|$ times. We can thus have that $C O S T_{\text {comp }} \leq\left(1+d_{\max }\right) \cdot\left|\bigcup_{j \in C C S\left(v_{i}\right)} T_{s}(j)\right| \cdot N \leq\left(1+d_{\max }\right) \cdot\left|C C S\left(v_{i}\right)\right| \cdot N \leq$ $\left(1+d_{\max }\right) \cdot d_{\max } \cdot N=O\left(N \cdot d_{\max }^{2}\right)$.

Accordingly, the total message complexity of our proposed DLBPA-A is $C O S T_{\text {init }}+C O S T_{\text {comp }} \leq O\left(N^{2}\right)+O\left(N \cdot d_{\text {max }}^{2}\right)=O\left(N^{2}+N \cdot d_{\text {max }}^{2}\right)$.

\section{Practical Issues}

In this section, we will discuss the practical issues faced when implementing our proposed solutions.

Note that, we make the same assumptions as most of the existing works about broadcast scheduling for low-duty-cycle WSNs, that is, the assumptions made in our paper are all commonly used in the existing related works and our solution does NOT bring any additional overhead compared with the existing related works. Actually, these commonly used assumptions will cost much less overhead in practice. For example, we only need to realize a local synchronization between neighboring nodes in our paper. In real WSNs, local synchronization can be achieved by using an existing high-efficient MAC-layer time stamping technique FTSP (Flooding Time Synchronization Protocol) [31], which achieves an accuracy of 2.24us with the cost of exchanging only a few bytes of packets among neighboring nodes every 15 minutes. Since the length of each time slot is usually long enough (at least tens of milliseconds) in practice, the accuracy of $2.24 \mathrm{us}$ is sufficient. Also, the assumption that each node is aware of the working schedules of all its neighboring nodes can be realized by 
only exchanging the schedules between neighboring nodes immediately after the deployment. In our solution, we will use a binary string to represent the working schedule, e.g., to use the binary string $<0010>$ to represent the periodic working schedule $\{2\}$ with $L=4$. In this way, we can find that the exchange cost of the working schedules between neighboring nodes is quite low especially when an efficient string compression scheme is adopted. More importantly, this exchange is only a one-time task during the implementation of our solution. Therefore, this assumption will bring much less overhead in practice.

In our centralized solution, each node will initially keep awake immediately after the deployment and the sink will derive the network topology according to some existing solution. Based on the network topology, the sink will execute our centralized algorithm to obtain the broadcasting schedule and then distribute it to all nodes in the network, and this will be done during the initialization phase of the network and is a one-time task. Actually, this is also the commonly used implementation for most of the existing centralized algorithms. For our distributed solution, we will first install our proposed distributed algorithm on each node, then each node will execute our distributed algorithm as described in Section 6.1 immediately after the deployment. For both solutions, once getting the transmission strategy, each node will put itself into the low-duty-cycle mode according to its own working schedule (the working schedule can be determined independently by itself or can rely on a particular power management protocol). Upon receiving a packet, any node $v$ will check its header to see whether it is a broadcast packet, if yes, node $v$ will forward this packet according to its own transmission strategy. In this paper, we do not need to require specific sink location. Note that our objective is to minimize the maximum transmission load of a broadcast schedule for low-duty-cycle WSNs, subject to the constraint that each node should have the minimum end-to-end delay under the broadcast schedule. We can easily find that the change of sink location will only influence the minimum end-to-end delay for each node, but will absolutely not influence the performance (e.g., approximation ratio) of our solution in terms of energy fairness. 
In this paper, we assume that our target applications will not experience a notable change on the link qualities, which implies the topology changes mainly come from the energy depletion of sensing nodes. In practice, some emerging technologies (e.g., Wireless Charging Technology [32] and Mobile Robot Technology [33].) can help us deal with such kind of topology changes. For example, we can set an energy threshold for each node, and any node will transmit an alarm packet to the sink once its residual energy is below this threshold. Upon receiving the alarm packet, the sink will send a mobile charger to the target node and wirelessly recharge it, or send a mobile robot there to replace the target node with a new one that has the same code and configuration as the target node. In this case, we do not need to consider the topology change and the network initialization phase is just a one-time task, which implies the control traffic in the network initialization phase will bring much less overhead compared with the long-term run of the broadcasting applications so that its cost can be approximately neglected.

\section{Performance Evaluation}

In this section, we evaluate the performance of our solutions via simulations.

\subsection{Experiment Setup}

In our setting, we consider that all sensor nodes are uniformly distributed in a $100 \mathrm{~m} * 100 \mathrm{~m}$ sensory field with the sink node located at the center of the field (i.e., $(50 \mathrm{~m}, 50 \mathrm{~m}))$. For simplicity and without loss of generality, we assume that one period of any node's working schedule contains only one active state time slot, and the disk communication model is adopted, i.e., any node $A$ can successfully deliver a packet to any node $B$ if and only if node $B$ is located within the communication range of node $A$. Actually, our solution can still be available and well-performed for a general communication model where the communication link does not totally depend on the distance. Here, we assume that all nodes have the same communication range $R_{c}$ and we set $R_{c}=10 \mathrm{~m}$. Further, we let each sensing node randomly determine its own working schedule 
and for the sink node $v_{0}$, we set $T_{s}\left(v_{0}\right)=\{0\}$. Unless otherwise stated, we set $N=800, L=50$ (i.e., duty cycle $=2 \%$ ), and all the results are obtained by averaging over 50 experiments.

As stated in Section 3.1, we assume the interarrival time between any two successive broadcast requests from the sink is more than the minimum broadcast latency, which implies we do not need to define specific broadcasting message traffic in our performance evaluation, since this practical assumption can make sure that the collision between any two requests must not occur under whatever broadcasting message traffic. Likewise, we also do not need to consider the control traffic, because the control traffic only occurs at the construction stage of broadcasting schedule, which means it is only a one-time task. Obviously, the control traffic, which only occurs at the initialization phase of the deployed network, will not influence the broadcasting message traffic in practice. As the control packets are short and their transmissions only occur at the initialization phase of the deployed network, the overhead induced by our proposed solutions are so small compared with the whole energy consumption that can be neglected. Thus we can only take into account the transmission load when computing energy consumption.

Here, we compare our solutions with two traditional solutions. One is the well-known Bellman-Ford Algorithm, which is commonly used to find the shortest path tree. Another one is the Random Parent Selection Algorithm (RPS-A), in which each sensing node $v_{i}$ will randomly select one node in $C P S\left(v_{i}\right)$ as its parent after performing MDPGC-A.

\subsection{Performance of Energy Fairness}

First, we will show the performance of energy fairness. As shown in [1], idle listening mode has nearly the same energy consumption rate with the receiving mode for each real sensor node in practice, which implies any node will consume nearly a constant energy amount in an active time slot no matter whether any packet is received in this slot. In other words, we do not need to consider the receiving energy consumption for all nodes (i.e., the receiving energy consump- 
tion for all nodes can be approximately neglected), because in our model, each node only receives the packet at its scheduled active time slot and the receiving of packets does not bring any additional energy consumption. In addition, we assume that our target networks have commonly adopted the load-balanced data collection protocols and also assume that each node has the same initial energy immediately after the deployment, therefore, we only need to consider transmission load as the main metric of energy consumption for broadcasting and similar transmission loads can absolutely ensure energy fairness.

Fig. 8(a) and Fig. 8(b) compare our solutions with the traditional solutions in terms of the maximum transmission load, and we find that our proposed LBPA-A and DLBPA-A can always get much better performance than Bellman-Ford Algorithm and RPS-A no matter how $N$ or $L$ (i.e., duty cycle) varies. As shown in both Fig. 8(a) and Fig. 8(b), Bellman-Ford Algorithm exhibits the worst performance since in Bellman-Ford Algorithm, each node $v_{i}$ will only select the first coming node in $C P S\left(v_{i}\right)$ as its parent which would make some nodes undertake much more load. In addition, LBPA-A exhibits a slightly better performance than DLBPA-A. This is because compared with DLBPAA, LBPA-A utilizes the global information to greedily reduce the redundancy and adopts a better comparison metric considering the influence of the removal of the redundant transmission on the RD values of the transmissions from the other senders. Also, we can find that under the assumption of uniform distribution and disk communication model, LBPA-A and DLBPA-A can always get a stable maximum transmission load, i.e., around 2-3, which is near-optimal as the optimal solution must be at least 1 .

Furthermore, as shown in Fig. 8(c), LBPA-A and DLBPA-A can always achieve a much lower Standard Deviation of the transmission load for all sensing nodes, and Fig. 8(d) exhibits a representative CDF of transmission load for these four solutions. Fig. 9 explicitly illustrates the distribution of transmission loads. We can find that LBPA-A and DLBPA-A exhibit a much better load balancing between sensor nodes. Accordingly, we can conclude that compared with the traditional Bellman-Ford Algorithm and RPS-A, our proposed LBPA-A and 


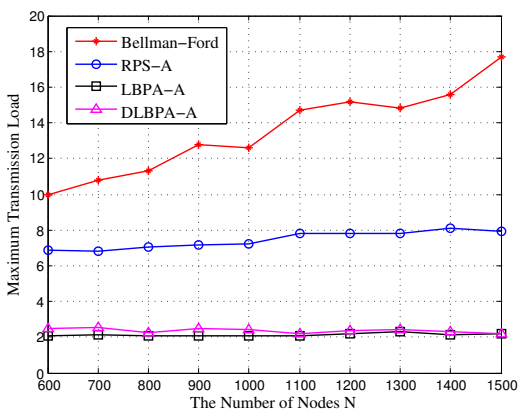

(a) maximum transmission load vs. $N$

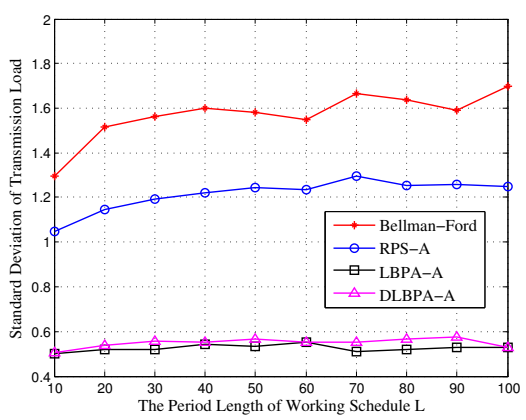

(c) standard deviation vs. $L$

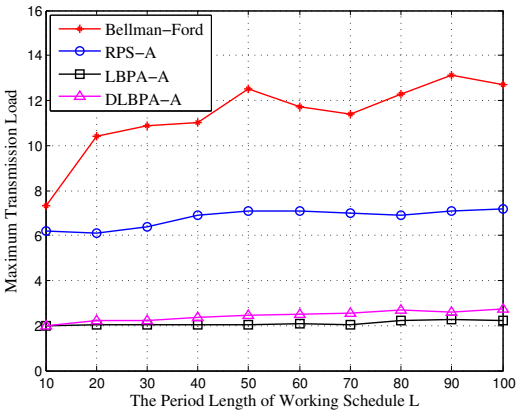

(b) maximum transmission load vs. $L$

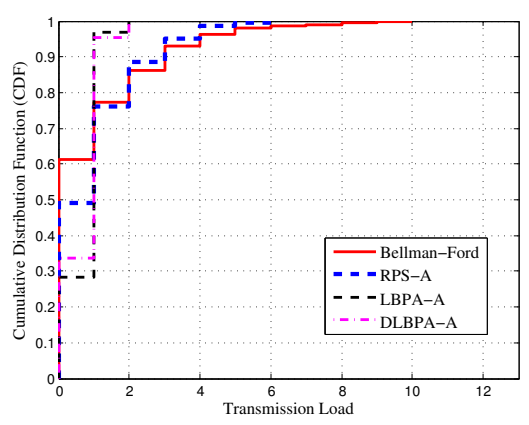

(d) CDF of transmission load

Figure 8: Performance of energy fairness

DLBPA-A can obtain a better energy fairness.

\subsection{Performance of Total Energy Consumption}

As the efficient schemes for redundant transmissions reduction are adopted, LBPA-A and DLBPA-A perform much better in terms of the total transmission load than Bellman-Ford Algorithm and RPS-A, which can be seen in Fig. 10(a) and Fig. 10(b). Here, the total transmission load denotes the transmission load cumulated on all nodes. In Fig. 10(a) and Fig. 10(b), we can find that DLBPAA performs slightly better than LBPA-A, and compared with Bellman-Ford Algorithm and RPS-A, the performance advantage of LBPA-A and DLBPA-A will be enlarged as the number of nodes $N$ increases or the period length of working schedule $L$ decreases (i.e., the duty cycle increases). This is because the increase of $N$ or the decrease of $L$ will make more neighboring nodes have the same working schedule, which would bring more redundant transmissions 


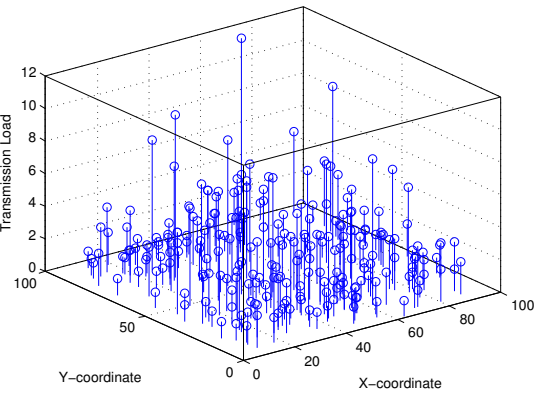

(a) Bellman-Ford

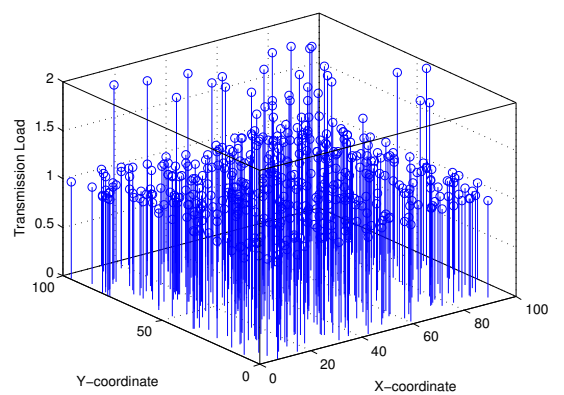

(c) LBPA-A

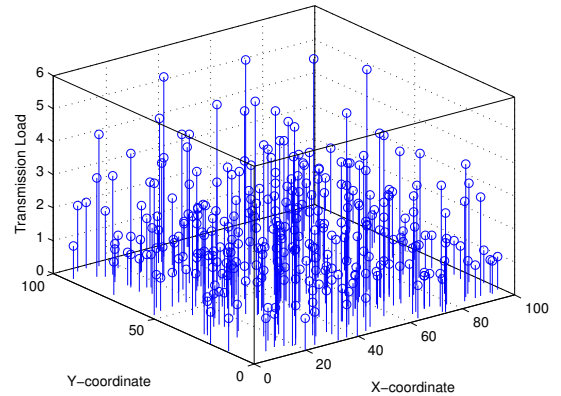

(b) RPS-A

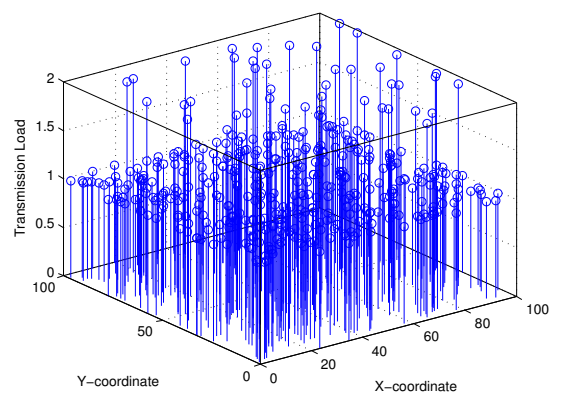

(d) DLBPA-A

Figure 9: Illustration of Transmission Load Distribution ( $N=600$ and $L=50)$.

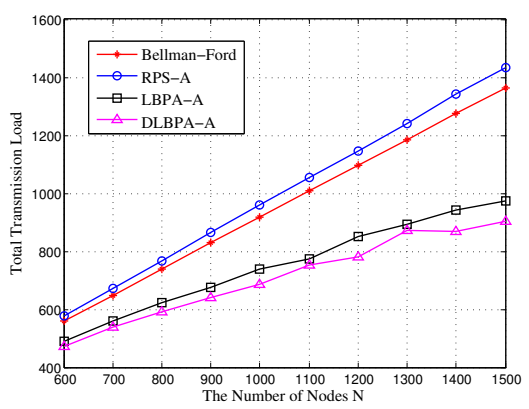

(a) total transmission load vs. $N$

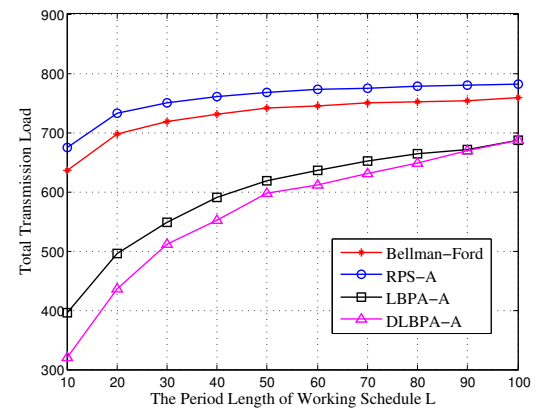

(b) total transmission load vs. $L$

Figure 10: Performance of total energy consumption

and provide more opportunities for LBPA-A and DLBPA-A to improve total energy consumption by efficiently reduce the redundancy of transmissions. 


\subsection{Performance of Delivery Ratio}

In the above sections, we do not consider the packet collision problem for simplicity. Here, we will mainly investigate the impact of packet collision problem on delivery ratio for our solutions in low-duty-cycle WSNs and propose a simple and efficient approach to further improve the delivery ratio in practice. First, we assume each time slot can only accommodate one packet transmission, i.e., $|T|=1$ where $|T|$ denotes the largest number of packet transmissions that each time slot can accommodate. Here, we define that a collision will happen if and only if some node receives the same packet from multiple different senders at the same time and once a collision happens at some node, the node will fail to receive this packet. Fig. 11(a) and Fig. 11(b) show the delivery ratio of our solutions and the traditional solutions when $|T|=1$. We can find that no matter how $N$ or $L$ (i.e., duty cycle) varies, LBPA-A and DLBPA-A can always achieve a much higher delivery ratio than Bellman-Ford Algorithm and RPS-A, and DLBPA-A performs better than LBPA-A. The delivery ratio of our solutions will become higher as $N$ decreases or $L$ increases (i.e., duty cycle decreases). This can be explained by the fact that the decrease of the number of nodes or the decrease of duty cycle will make less neighboring nodes have the same working schedule, which would reduce the collision chance in the network. As shown in Fig. 11(a), LBPA-A and DLBPA-A can always keep a delivery ratio of over $80 \%$ when $N$ varies from 600 to 1500 and a delivery ratio of over $90 \%$ can be achieved when the network scale is moderate (i.e., $N \leq 1000$ ). Specifically, the delivery ratio of LBPA-A and DLBPA-A can respectively achieve $95.56 \%$ and $97.29 \%$ when $N=600$. As in Fig. 11(b), LBPA-A and DLBPA-A can achieve a delivery ratio of over $90 \%$ when $L \geq 50$ (i.e., duty cycle $\leq 2 \%$ ). Specifically, the delivery ratio of LBPA-A and DLBPA-A can respectively achieve $96.85 \%$ and $98.7 \%$ when $L=100$ (i.e., duty cycle=1\%). We thus conclude that for moderate-scale low-duty-cycle WSNs, our solutions can always achieve a considerable delivery ratio when $|T|=1$.

In practice, each time slot is always set as long enough (i.e., $|T|>1$ ), because in the applications of data collection, each forwarder is usually expected to 


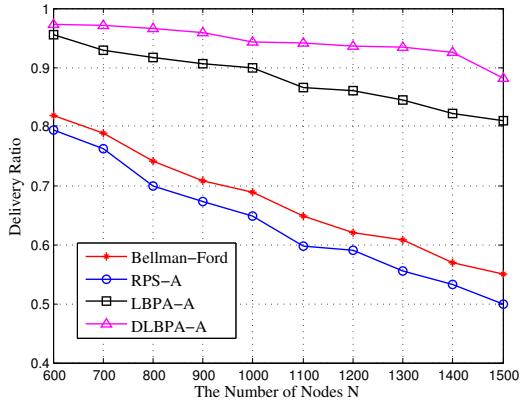

(a) delivery ratio vs. $N$

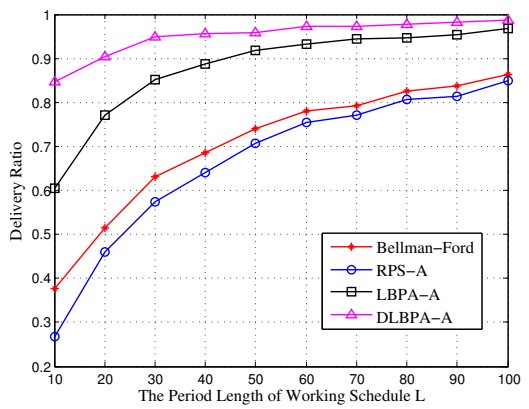

(b) delivery ratio vs. $L$

Figure 11: Performance of delivery ratio when $|T|=1$

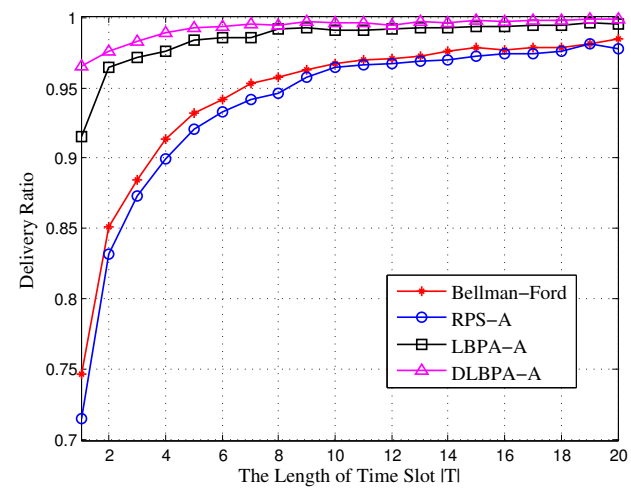

Figure 12: Relationship between delivery ratio and $|T|$

receive as more data packets as possible from its children at one time slot to make the data aggregation in order to achieve high energy efficiency. Here, we utilize a simple and efficient random transmission strategy to further improve the delivery ratio of our solutions. Specifically, we will divide each time slot into $|T|$ equal time units so that each time unit can just accommodate one packet transmission, and if any node is scheduled to transmit a broadcasting packet at some time slot, it will randomly select a time unit of this time slot to send the packet. By this way, the packet collision chance can be further reduced greatly. Fig. 12 exhibits the relationship between $|T|$ and delivery ratio for all four solutions when random transmission strategy is adopted. We can find that the delivery ratio increases as $|T|$ increases for all four solutions and 
our solutions always exhibit better performance than the traditional solutions regardless of $|T|$, and the delivery ratio of our solutions will be significantly improved especially when $|T|$ changes from 1 to 2 . In Fig. 12 where $N=800$, $L=50$ and $R_{c}=10 \mathrm{~m}$, it can be seen that when $|T|>1$, both of LBPA-A and DLBPA-A always achieve a delivery ratio of over $95 \%$, and a delivery ratio of over $99 \%$ can be achieved when $|T| \geq 8$ for LBPA-A and $|T| \geq 5$ for DLBPAA, which indicates that for low-duty-cycle WSNs with random transmission strategy, the packet collision problem can thus be approximately neglected as the delivery ratio usually approaches to $100 \%$ in practice.

As the interarrival time between any two successive broadcast requests is always far more than the minimum broadcast latency in real WSNs, the delivery ratio of our proposed solutions will thus absolutely not be influenced under whatever broadcasting message traffic. Intuitively, the topology change will influence the delivery ratio of broadcasting, however, it is worthless to consider the influence of topology change on the delivery ratio. This is because we can periodically re-execute our algorithm to update the broadcasting schedule in our solutions according to the history record about the frequency of topology change and thus the influence of topology change on the delivery ratio is so less that it can be neglected in practice. Moreover, it does not make any sense to characterize the relationship between topology change and delivery ratio, since we can easily find that some local link or node change could increase, decrease or unchange the delivery ratio and there does not exist a notable correlation between topology change and delivery ratio.

\section{Conclusion}

In this paper, we mainly focus on the Load-Balanced Minimum Delay Broadcast Scheduling Problem (LB-MDBS) for low-duty-cycle WSNs. We first transform our LB-MDBS problem into the equivalent Load-Balanced Parents Assignment Problem (LBPA), and prove its NP-hardness. Then, we address this problem by proposing the Load-Balanced Parents Assignment Algorithm (LBPAA) which achieves $\lambda$-approximation, where $\lambda$ denotes the maximum number of 
neighbors that are scheduled to wake up at the same time and is typically a small number in low-duty-cycle WSNs. Also, we present an efficient distributed solution. Finally, the high-efficiency of our solutions has been evaluated by simulations in terms of energy fairness, total energy consumption and delivery ratio.

\section{Acknowledgement}

The work is partly supported by China 973 project (2012CB316200) and China NSF grants (61133006, 61021062, 60903179).

\section{References}

[1] M. Stemm and R.H. Katz. Measuring and reducing energy consumption of network interfaces in hand-held devices. IEICE Trans. Commun., vol. E80-B, no. 8, pp. 1125-1131, 1997.

[2] Texas Intruments. 2.4 GHz IEEE 802.15.4 / ZigBee-Ready RF Transceiver (Rev. C), 2013. Available at http://www.ti.com/lit/ds/symlink/cc2420.pdf.

[3] F. Wang and J. Liu. On reliable broadcast in low duty-cycle wireless sensor networks. IEEE Transactions on Mobile Computing, 11(5): 767-779, 2012.

[4] J. Hong, J. Cao, W. Li, S. Lu, and D. Chen. Minimum-transmission broadcast in uncoordinated duty-cycled wireless ad hoc networks. IEEE Trans. Veh. Technol., vol. 59, no. 1, pp. 307-318, 2010.

[5] S. Guo, S. Kim, T. Zhu, Y. Gu, T. He. Correlated flooding in low-duty-cycle wireless sensor networks. In ICNP, 2011.

[6] Y. Sun, O. Gurewitz, S. Du, L. Tang, and D.B. Johnson. ADB: an efficient multihop broadcast protocol based on asynchronous duty-cycling in wireless sensor networks. In ACM SenSys, pages 43-56, 2009.

[7] L. Xu, J. Cao, S. Lin, H. Dai, X. Wu, G. Chen. Energy-efficient broadcast scheduling with minimum latency for low-duty-cycle wireless sensor networks. In Proc. of IEEE MASS'13, 2013. 
[8] S. Lai, B. Ravindran. On Multihop Broadcast over Adaptively Duty-Cycled Wireless Sensor Networks. In DCOSS'10, 2010.

[9] T. Zhu, Z. Zhong, T. He, Z. Zhang. Exploring link correlation for efficient flooding in wireless sensor networks. In NSDI, 2010.

[10] K. Han, Y. Liu, J. Luo. Duty-cycle-aware minimum-energy multicasting in wireless sensor networks. IEEE/ACM Transactions on Networking (TON), 2013, 21(3): 910-923.

[11] K. Han, L. Xiang, J. Luo, et al. Energy-efficient reliable data dissemination in duty-cycled wireless sensor networks. In proceedings of the fourteenth ACM international symposium on Mobile ad hoc networking and computing. ACM, 2013: 287-292.

[12] L. Cheng, Y. Gu, T. He, et al. Dynamic switching-based reliable flooding in low-duty-cycle wireless sensor networks. In Proceedings of IEEE INFOCOM. 2013: 1393-1401.

[13] J. Niu, L. Cheng, Y. Gu, J.H. Jun, Q. Zhang. Minimum-Delay and EnergyEfficient Flooding Tree in Asynchronous Low-Duty-Cycle Wireless Sensor Networks. In Proc. of IEEE WCNC, 2013.

[14] F. Ferarri, M. Zimmerling, L. Thiele and O. Saukh. Efficient Network Flooding and Time Synchronization with Glossy. In Proc. of ACM IPSN'11, 2011.

[15] 10 X. Wu, G. Chen and S.K. Das. Avoiding energy holes in wireless sensor networks with nonuniform node distribution. IEEE Transactions on Parallel and Distributed Systems (TPDS), vol. 19, No. 5, May 2008.

[16] R. Jurdak, P. Baldi and C.V. Lopes. Adaptive Low Power Listening for Wireless Sensor Networks. IEEE Transactions on Mobile Computing, Volume 6 Issue 8, pages 988-1004, 2007.

[17] S. Xiong, J. Li, M. Li, J. Wang, and Y. Liu. Multiple task scheduling for low-duty-cycled wireless sensor networks. In Proc. of IEEE INFOCOM'11, pp. 1323-1331, 2011. 
[18] Z. Li, Y. Peng, D. Qiao and W. Zhang. LBA: Lifetime balanced data aggregation in low duty cycle sensor networks. In Proc. of IEEE INFOCOM'12, pp. 1844-1852, 2012.

[19] L. Mai, L. Shangguan, J. Du, Z. Li, and M. Li. Load Balanced Rendezvous Data Collection in Mobile Wireless Sensor Networks. In Proc. of IEEE MASS'11, 2011.

[20] Z. Li, M. Li, Y. Liu. Towards energy-fairness in asynchronous duty-cycling sensor networks. ACM Transactions on Sensor Networks (TOSN), 2014, 10(3): 38 .

[21] L. Xu, G. Chen, X. Yin, P. Yang, B. Yang. LORP: A Load-Balancing Based Optimal Routing Protocol for Sensor Networks with Bottlenecks. In Proc. of IEEE WCNC'09, 2009.

[22] X. Zhu, X. Wu, G. Chen. An exact algorithm for maximum lifetime data gathering tree without aggregation in wireless sensor networks. Wireless Networks, 2015, 21(1): 281-295.

[23] Q. Cao, T. Abdelzaher, T. He, and J. Stankovic. Towards Optimal Sleep Scheduling in Sensor Networks for Rare Event Detection. In IPSN05, 2005.

[24] Y. Gu and T. He. Data forwarding in extremely low duty-cycle sensor networks with unreliable communication links. In Proc. of ACM SenSys'07, pp. 321-334, 2007.

[25] S. Guo, Y. Gu, B. Jiang, and T. He. Opportunistic Flooding in Low-DutyCycle Wireless Sensor Networks with Unreliable Links. In MobiCom'09, 2009.

[26] X. Jiao, W. Lou, J. Ma, J. Cao, X. Wang, X. Zhou. Duty-cycle-aware minimum latency broadcast scheduling in multi-hop wireless networks. In ICDCS, 2010. 
[27] L. Su, B. Ding, Y. Yang, T.F. Abdelzaher, G. Cao, and J. Hou, ocast: Optimal multicast routing protocol for wireless sensor networks. In ICNP, pages 151-160, 2009.

[28] M.R. Garey, D.S. Johnson. Computers and Intractability: A guide to the theory of NP-completeness. W.H. Freeman and Co., 1979.

[29] N.J. Harvey, R.E. Ladner, L. Lovasz and T. Tamir. Semi-matchings for bipartite graphs and load balancing. In WADS, 2003.

[30] J. Fakcharoenphol, B. Laekhanukit, and D. Nanongkai. Faster algorithms for semi-matching problems. In ICALP, 2010.

[31] M. Marti, B. Kusy, G. Simon, and A. Ldeczi. The Flooding Time Synchronization Protocol. In Proc. of ACM SenSys'04, 2004.

[32] H. Dai, X. Wu, G. Chen, L. Xu and S. Lin. Minimizing the number of mobile chargers for large-scale wireless rechargeable sensor networks. Computer Communications, 2014, 46: 54-65.

[33] G. Fletcher, X. Li, A. Nayak, et al. Randomized robot-assisted relocation of sensors for coverage repair in wireless sensor networks. Vehicular Technology Conference Fall (VTC 2010-Fall), 2010 IEEE 72nd. IEEE, 2010: 1-5. 